DEPARTMENT OF ECONOMICS

Working Paper Series

Measuring risk aversion with lists: A new bias

Antoni Bosch-Domenech

Universitat Pompeu Fabra

Joaquim Silvestre

U.C. Davis

September 26, 2012

Paper \# 12-23

Various experimental procedures aimed at measuring individual risk aversion involve a list of pairs of alternative prospects. We first study the widely used method by Holt and Laury (2002), for which we find that the removal of some items from the lists yields a systematic decrease in risk aversion and scrambles the ranking of individuals by risk aversion. This bias, that we call embedding bias, is quite distinct from other confounds that have been previously observed in the use of the Holt and Laury method. It may be related to empirical phenomena and theoretical developments where better prospects increase risk aversion. Nevertheless, we also find that the more recent elicitation method due to Abdellaoui et al. (2011), also based on lists but using only one and the same probability in the list, does not display any statistically significant bias when the corresponding items of the list are removed. Our results suggest that methods other than the popular Holt and Laury one may be preferable for the measurement of risk aversion.

Department of Economics

One Shields Avenue

Davis, CA 95616

(530)752-0741

http://www.econ.ucdavis.edu/working_search.cfm 
Measuring risk aversion with lists: A new bias

\author{
by \\ Antoni Bosch-Domènech \\ antoni.bosch@upf.edu \\ Universitat Pompeu Fabra \\ and \\ Joaquim Silvestre \\ jbsilvestre@ucdavis.edu \\ University of California, Davis
}

Forthcoming in Theory and Decision, Manuscript Number THEO1357R1 


\title{
Measuring risk aversion with lists: A new bias
}

\begin{abstract}
Various experimental procedures aimed at measuring individual risk aversion involve a list of pairs of alternative prospects. We first study the widely used method by Holt and Laury (2002), for which we find that the removal of some items from the lists yields a systematic decrease in risk aversion and scrambles the ranking of individuals by risk aversion. This bias, that we call embedding bias, is quite distinct from other confounds that have been previously observed in the use of the Holt and Laury method. It may be related to empirical phenomena and theoretical developments where better prospects increase risk aversion. Nevertheless, we also find that the more recent elicitation method due to Abdellaoui et al. (2011), also based on lists but using only one and the same probability in the list, does not display any statistically significant bias when the corresponding items of the list are removed. Our results suggest that methods other than the popular Holt and Laury one may be preferable for the measurement of risk aversion.
\end{abstract}

KEY WORDS: Risk aversion, risk attitudes, experiments, lists, elicitation method, Holt, Laury, Abdellaoui, Driouchi, l’Haridon, independence axiom, probability weighting. JEL Classification Number: C 91 


\section{Measuring risk aversion with lists: A new bias *}

\section{Introduction}

Various experimental procedures aimed at eliciting information on risk attitudes involve a list of pairs of alternative prospects. The present paper investigates the robustness of such procedures with respect to the removal of some pairs from the list.

Specifically, we study the widely used method by Holt and Laury (2002) [HL in what follows] and the new method proposed by Abdellaoui et al. (2011) [ADH in what follows], also based on lists of pairs of prospects, and enquire whether the removal of some pairs from their list affects the choices made by experimental subjects. ${ }^{1}$ While having many things in common, both methods differ substantively. Following Farquhar (1984) classification, the HL procedure can be described as a probability equivalence method, while the ADH procedure can be considered a certainty equivalence method.

Both methods for assessing individual risk are subject to several biases discussed in the literature (see Hershey and Schoemaker, 1985). In particular, any probability equivalence method measures risk preferences by means of a probability scale, which may distort the results due to probability weighting when subjects have non-expected utility preferences (e. g., Wakker and Deneffe, 1996, Drichoutis and Lusk, 2012, Fehr-Duda and Epper, 2012). ${ }^{2}$ In fact, Fehr-Duda et al., (2010) show that the probability weighting function itself may vary with the amounts at stake.

On the other hand, if a certainty equivalence method is used, a certainty effect (Hershey et al., 1982, McCord and Neufville, 1986) may bias the risk assessment towards more risk aversion whenever subjects have non-expected utility preferences. Both the HL and ADH procedures, in addition, may suffer from the disadvantages of multiple-price lists (Andersen et al., 2006): multiple-price lists create a frame that may bias the experimental subjects towards the middle of the list.

\footnotetext{
* We acknowledge financial support from the Spanish Ministerio de Educación y Ciencia under research project SAJ2005-03891/ECON. We have benefited from an excellent referee report, and we are indebted to our colleagues Michael Greenacre and Albert Satorra for help in the statistical analyses.

${ }^{1}$ According to Drichoutis and Lusk (2012), Holt and Laury (2002) is the third most highly cited paper published by the American Economic Review and their work has been cited more than 1,500 times according to Google scholar.

${ }^{2}$ Bruhin et al. (2010) estimate that "roughly $80 \%$ of the subjects [in three different experimental data sets] exhibit significant deviations from linear probability weighting."
} 
It has already been noted that, in the HL procedure, the order in which the treatments are implemented may confound the results (Harrison et al., 2005; see also Holt and Laury, 2005). Andersen et al. (2006) modify the standard HL list and "break its symmetry" by constructing two skewed lists, which they call skewHI and skewLO. They observe that while skewHI yields the same estimated coefficients of relative risk aversion, as does skewLO when it breaks the symmetry of the standard HL list, skewLO reduces the estimated coefficient for two variations of the HL method. Dave et al. (2010) consider the advantages and disadvantages of differing degrees of difficulty depending on the characteristics of the subjects. Lévy-Garboua et al. (2012) test the effects of simultaneous vs. sequential decisions, as well as of order, on the frequency of inconsistent behaviors. Beauchamp et al. (2012) find that manipulating the intermediate sure payoff in a list robustly changes measured risk preferences. Other framing effects are reported in Isaac and James (2000).

In this paper we observe a new bias that we uncover by removing some pairs at the beginning and/or the end of the lists presented to the experimental subjects. We first consider the HL procedure: we find that the removal of better, end-of-list, pairs reduces the frequency of riskaverse choices relative to the whole list. The observed dependence, within subjects, of the risk attitude in a particular pair of prospects on the list where the pair is embedded violates the independence axiom. It follows that the expected utility hypothesis is violated, and hence Holt and Laury's (2002) numerical estimation of the degree of risk aversion based on von NeumannMorgenstern (vNM) utility functions is invalid. At the more fundamental level, we also find that subjects may switch between risk aversion and attraction in a particular pair depending on the list in which the pair is embedded: different subjects may switch in different directions, therefore failing to preserve any individual rankings by risk aversion.

Since decisions depend on how the different prospects are embedded in a list, we call this confound an embedding bias. This confound is quite distinct from the ones reported in the abovementioned literature. We, in particular, find that the way the list is ordered plays no role, and find no evidence of middle-of-the-list effects.

The embedding bias, together with other findings in the literature with the same flavor, may suggest a more general idea that the inclusion of better prospects in a list of choices favors risk aversion. But it cannot be a universal principle. We conduct similar robustness checks on the ADH 
procedure and find no evidence of such a bias: the frequency of risk-averse choices for a given pair of the list is statistically invariant with respect to the deletion of other items. ${ }^{3}$

Our results on the embedding bias are cast in terms of the frequency of risk-averse choices, and hence they are model independent: they do not explicitly or implicitly rest on assumptions often postulated in quantifying risk aversion (such as expected utility, or, equivalently, the independence axiom, or particular hypotheses on non-expected utility), not to mention specific functional forms for utility, value or probability weighting functions.

\section{The Holt \& Laury method}

\subsection{Purpose}

Holt and Laury state (2002, p. 1645) that they "present subjects with a menu of choices that permits measurement of the degree of risk aversion, and also estimation of its functional form.” In order to measure the degree of risk aversion, they first match (Table 3, p. 1649) the subjects' choices to risk aversion intervals based on the CRRA (constant relative risk aversion) vNM utility function $x^{1-r} /(1-r)$ (the variable $x$ is the ex post amount of money, and the parameter $r$ is the coefficient of relative risk aversion). The last pages of the paper report maximum-likelihood estimates of the parameters $r$ and $\alpha$ of the vNM function $\left(1-\exp \left(-\alpha x^{1-r}\right) / \alpha\right.$, first proposed by Saha (1993).

\subsection{Description of the Holt and Laury method}

Subjects in HL (2002) face a list of ten pairs of binary lotteries, which we can number from one to ten as in Table 1 below, each pair involving a "safe” lottery $(S)$ and a "risky" one $(R)$. The terms “safe” and “risky,” used by HL (2002), must be understood in a loose sense and relative to each other: in a given pair, lottery $R$ gives a larger good payoff, but a lower bad payoff, than $S .{ }^{4}$ The two lotteries in a pair entail the same probabilities for the good and bad payoffs, and their expected

\footnotetext{
${ }^{3}$ Also, our own method (Bosch-Domènech and Silvestre, 1999, 2006a, b, c, 2010) for eliciting risk attitudes does not display a statistically significant embedding effect. Our method shares two features with ADH: one of the options in each pair is a certain amount of money, and the probabilities of the other option, a nondegenerate lottery, are fixed. One difference between our method and ADH's one is that we change the quantities of both the certain option and the nondegenerate lottery in each pair and keep actuarial fairness between them, while in the ADH method only the quantities of the certain option vary.

${ }^{4}$ The lotteries were labeled $A$ and $B$, instead of $S$ and $R$, for the experimental subjects.
} 
values are different, i. e., $R$ is not a mean-preserving spread of $S$. Consequently, HL (2002) does not test for "strong” risk aversion which, except under special assumptions, is a more restrictive condition than (weak) risk aversion, see, e. g., Wakker (2010, pp. 75-76).

All $S$ lotteries offer the same payoffs, namely $\$ 2$ and $\$ 1.60$, but with varying probabilities. An $S$ lottery with a high lottery-pair number first-order stochastically dominates any $S$ lottery with a lower number, since it gives the larger payoff (\$2) with higher probability. The list of $R$ lotteries displays exactly the same feature. Hence, a lottery pair with a higher number offers an unambiguously better prospect than one with a lower number.

Of course, first-order stochastic dominance implies higher expected value. The last three columns in Table 1 (not shown to the experimental subjects) indicate the expected dollar values of the safe lottery in the pair (denoted $E V^{S}$ ) and that of the risky lottery (denoted $E V^{R}$ ), as well as the difference between the two. A risk-neutral individual would select the compound choice $S S S S / R R R R R R$. Thus, a subject who selects SSSSS/RRRRR displays risk aversion.

\subsection{Our experimental design: Changing list length in Holt and Laury}

Design. We designed five treatments, numbered 1 to 5, and carried them out in six sessions, labeled $A$ to $F$. Our Treatment 1 is the control treatment, where subjects face the complete list of Table 1, with euro payoffs obtained by multiplying by three the dollar amounts of Table 1 . These payoffs were maintained in all treatments.

In treatments 2 to 5 we ran the experiment with lists of seven (lottery) pairs where some of the better pairs and/or some of the worse pairs of Table 1 (three in total) have been eliminated.

Specifically, in Treatment 2, each subject faced the seven-pair list obtained by deleting the first three rows of Table 1. In Treatment 3, each subject faced the seven-pair list obtained by deleting rows 1, 2 and 10 of Table 1 . In Treatment 4, each subject faced the seven-pair list obtained by deleting rows 1, 9 and 10 of Table 1. Finally, in Treatment 5 each subject faced the seven-pair list described in Table 2 and obtained by deleting the last three rows from Table 1. We were particularly interested in the decisions for pairs 4 to 7 , which are present in all five treatments. 


\begin{tabular}{|c|c|c|c|c|c|c|c|c|c|c|c|}
\hline \multirow[b]{2}{*}{$\begin{array}{l}\text { Lottery } \\
\text { Pair \# }\end{array}$} & \multicolumn{4}{|c|}{ Safe Lottery $(S)$} & \multicolumn{4}{|c|}{ Risky Lottery $(R)$} & \multirow[b]{2}{*}{$E V^{S}$} & \multirow[b]{2}{*}{$E V^{R}$} & \multirow[b]{2}{*}{ Difference } \\
\hline & Prob. & Payoff & Prob. & Payoff & Prob. & Payoff & Prob. & Payoff & & & \\
\hline 1 & 0.1 & $\$ 2$ & 0.9 & $\$ 1.60$ & 0.1 & $\$ 3.85$ & 0.9 & $\$ 0.10$ & $\$ 1.64$ & $\$ 0.48$ & $\$ 1.17$ \\
\hline 2 & 0.2 & $\$ 2$ & 0.8 & 1.60 & 0.2 & $\$ 3.85$ & 0. & 0 & 1.68 & $\$ 0$ & $\$ 0.83$ \\
\hline 3 & 0.3 & $\$ 2$ & 0.7 & $\$ 1$. & 0.3 & $\$ 3$. & 0. & $\$$ & $\$ 1.72$ & $\$ 1$. & $\$ 0.49$ \\
\hline 4 & 0.4 & $\$ 2$ & 0.6 & $\$ 1.60$ & 0.4 & $\$ 3.85$ & 0.6 & $\$ 0.10$ & $\$ 1.76$ & $\$ 1.60$ & $\$ 0.16$ \\
\hline 5 & 0.5 & $\$ 2$ & 0.5 & $\$ 1.60$ & 0.5 & $\$ 3.85$ & 0.5 & $\$ 0.10$ & $\$ 1.80$ & $\$ 1.98$ & $-\$ 0.17$ \\
\hline 6 & 0.6 & $\$ 2$ & 0.4 & $\$ 1$. & 0.6 & $\$ 3$ & 0. & $\$ 0$ & $\$$ & $\$ 2.35$ & $-\$ 0.51$ \\
\hline 7 & 0.7 & $\$ 2$ & 0.3 & $\$ 1.60$ & 0.7 & $\$ 3.85$ & 0.3 & $\$ 0.10$ & $\$ 1.88$ & $\$ 2.73$ & $-\$ 0.84$ \\
\hline 8 & 0.8 & $\$ 2$ & 0.2 & $\$ 1.60$ & 0.8 & $\$ 3.85$ & 0.2 & $\$ 0.10$ & $\$ 1.92$ & $\$ 3.10$ & $-\$ 1.18$ \\
\hline 9 & 0.9 & $\$ 2$ & 0.1 & $\$ 1.60$ & 0.9 & $\$ 3.85$ & 0.1 & $\$ 0.10$ & $\$ 1.96$ & $\$ 3.48$ & $-\$ 1.52$ \\
\hline 10 & 1 & $\$ 2$ & 0 & $\$ 1.60$ & 1 & $\$ 3.85$ & 0 & $\$ 0.10$ & $\$ 2.00$ & $\$ 3.85$ & $-\$ 1.85$ \\
\hline
\end{tabular}

Table 1. Design of the Holt and Laury (2002) experiments (adapted from Harrison et al., 2005).

\begin{tabular}{|c|c|c|c|c|c|c|c|c|c|c|c|}
\hline \multirow{2}{*}{$\begin{array}{l}\text { Lottery } \\
\text { Pair \# } \\
\end{array}$} & \multicolumn{4}{|c|}{ Safe Lottery $(S)$} & \multicolumn{4}{|c|}{ Risky Lottery $(R)$} & \multirow[b]{2}{*}{$E V^{S}$} & \multirow[b]{2}{*}{$E V^{R}$} & \multirow[b]{2}{*}{ Differenc } \\
\hline & Prob. & Payoff & Prob. & Payoff & Prob. & Payoff & Prob. & Payoff & & & \\
\hline 1 & 0.1 & $\$ 2$ & 0.9 & $\$ 1.60$ & 0.1 & $\$ 3.85$ & 0.9 & $\$ 0.10$ & $\$ 1.64$ & $\$ 0.48$ & $\$ 1.17$ \\
\hline 2 & 0.2 & $\$ 2$ & 0.8 & $\$ 1.60$ & 0.2 & $\$ 3.85$ & 0.8 & $\$ 0.10$ & $\$ 1.68$ & $\$ 0.85$ & $\$ 0.83$ \\
\hline 3 & 0.3 & $\$ 2$ & 0.7 & $\$ 1.60$ & 0.3 & $\$ 3.85$ & 0.7 & $\$ 0.10$ & $\$ 1.72$ & $\$ 1.23$ & $\$ 0.49$ \\
\hline 4 & 0.4 & $\$ 2$ & 0.6 & $\$ 1.60$ & 0.4 & $\$ 3.85$ & 0.6 & $\$ 0.10$ & $\$ 1.76$ & $\$ 1.60$ & $\$ 0.16$ \\
\hline 5 & 0.5 & $\$ 2$ & 0.5 & $\$ 1.60$ & 0.5 & $\$ 3.85$ & 0.5 & $\$ 0.10$ & $\$ 1.80$ & $\$ 1.98$ & $-\$ 0.17$ \\
\hline 6 & 0.6 & $\$ 2$ & 0.4 & $\$ 1.60$ & 0.6 & $\$ 3.85$ & 0.4 & $\$ 0.10$ & $\$ 1.84$ & $\$ 2.35$ & $-\$ 0.51$ \\
\hline 7 & 0.7 & $\$ 2$ & 0.3 & $\$ 1.60$ & 0.7 & $\$ 3.85$ & 0.3 & $\$ 0.10$ & $\$ 1.88$ & $\$ 2.73$ & $-\$ 0.84$ \\
\hline & & & & $\$ 100$ & مـ & $\$ 205$ & 2حم & $\$ 010$ & $\$ 102$ & $\$ 210$ & $\$ 118$ \\
\hline 10 & & 42 & & ..00 & & (1) & & & & & \\
\hline
\end{tabular}

Table 2. The deletion of the last three rows of Table 1.

Because of concern for the possible influence of the order in which the treatments are performed, we scrambled them and repeated one as a "return to baseline.” For instance, Treatment 1 preceded Treatment 5 three times while followed it four times. In each session, we ran four different treatments in the following orders.

Session A, with 28 subjects, implemented treatments 5, 3, 2, 1, 5;

Session $B$, with 24 subjects, treatments 2, 4, 5, 1, 2; 
Session $C$ with 21 subjects, treatments $1,5,3,2,1$;

Session $D$, with 24 subjects, treatments 3, 5, 2, 1, 3;

Session $E$, with 22 subjects, treatments 4, 1, 2, 3, 4;

and Session $F$, with 26 subjects, treatments 1, 4, 2, 5, 4.

Subjects. The subjects (totaling 145) were undergraduate students from Universitat Pompeu Fabra who volunteered.

Procedure. All the sessions were run manually. After the instructions were read and all questions answered, each subject in a session received ten pages (or seven, depending on the treatment) stapled together, each page containing one of the ten (or seven) lottery pairs corresponding to the first treatment in the session. As indicated above, the two lotteries in each pair, safe and risky, were called $A$ and $B$ in the experiment. Once the subjects had chosen either $A$ or $B$ in each page of the first treatment, the set of pages was collected and the next set of pages in the session was provided. The session finished when all five sets of stapled pages in the session had been completed by the subjects.

Earnings. Subjects were told that in order to compute their earnings at the end of the session, each of them individually would randomly select two of the five sets by drawing two numbers from an urn containing five numbers. Next, one pair in each of the two selected sets would be randomly chosen again by the subject herself (i. e., himself or herself) by drawing, for each of the two sets, one number from an urn containing as many numbers as pairs in the set (7 or 10). Finally, by means of a 10-sided die, the lotteries of the two selected pairs would be realized sequentially, and the subject would be paid according to her choices of $A$ or $B$ in each lottery pair. The sessions lasted about 45 minutes, but the individual payment procedure took an additional 30 minutes for some subjects.

\subsection{Including better (end-of-list) pairs favors risk aversion}

Tables A1 to A6 in Appendix A present the raw experimental data for sessions A to F. We can visualize the overall outcomes in Table 3, which displays the rate of safe choices per pair and treatment aggregated over Sessions $A$ to $F .^{5}$

\footnotetext{
${ }^{5}$ As is common when applying the HL method, we disregard the compound choices of the few subjects who switch to $S$ after having already chosen $R$ in a pair, i. e., of the form (...RS...). These compound choices can be shown to be inconsistent in the sense of being first-order stochastically dominated by the compound choice obtained by replacing the $R S$ pair by $S R$.
} 
Formally, and ignoring for the moment the last two columns, the entry in Table 3 for Pair $j(j=1$, $\ldots, 10)$ and Treatment $i(i=1, \ldots, 5)$ gives the aggregate rate of safe choices, defined as the quotient:

Number of $S$ choices in Pair $j$ and Treatment $i$ aggregated over Sessions $A-F$

Number of choices $(S$ and $R$ ) in Pair $j$ and Treatment $i$ aggregated over Sessions A-F

We have marked in boldface the pairs common to all five treatments, namely pairs 4 to 7 . Figure 1 plots the frequency of safe choices for treatments 1, 2 and 5. Recall that Treatment 1, our control, covers the ten pairs of Table 1 and Treatment 2 deletes the first three pairs, whereas Treatment 5 deletes the last three, as shown in Table 2.

By comparing the first two columns of Table 3 (Treatment 2 vs. control), we observe that the deletion of the first three (worse) pairs slightly increases the rate of safe choices. Treatment 5, which deletes the three last (best) pairs, shows a marked decrease in the rate of safe choices. See Figure 1, where the gap between the graphs for treatments 1 and 2 is dwarfed by the one between treatments 1 and 5. It appears that, as good pairs (bottom of list) are replaced by bad ones (top of list), risk aversion becomes less frequent.

These observations are confirmed by a Wald-type test for the equality of the rates of safe choices for pairs 4, 5, 6 and 7, where the switching occurs, in treatments 1,2 and $5 .{ }^{6}$ For treatments 1 and 2, the $p$-value of the Wald test equals 0.641 , not rejecting equality at the $5 \%$ level. For treatments 1 and 5, the corresponding $p$-value is 0.001 , rejecting equality. And for treatments 2 and 5 , the $p$-value is 0.000 , again rejecting equality.

Next, we focus on the individual decisions by each participant as she may confront treatments 1, 2 or 5 in the same session, testing whether any observed differences could be due to chance. To that effect, we use the McNemar test and obtain significant $p$-values (0.007 for pair 6, and 0.001 for pair 7) when comparing treatments 1 and 5, even after applying the Bonferroni

${ }^{6}$ The formula for the test statistic is $T=\left\|\frac{1}{\sqrt{x \cdot(1-x) /\left(n_{i}+n_{j}\right)}}\left(x_{i}-x_{j}\right)\right\|^{2}$, where $\|\cdot\|$ denotes the Euclidean norm, $x=\frac{1}{n_{i}+n_{j}}\left(n_{i} x_{i}+n_{j} x_{j}\right), x_{k}$ is the 4-dimensional vector of rates, and $n_{k}$ is the sample size, in Treatment $k, k=i, j$. 
correction. On the other hand, the $p$-values were not significant when comparing treatments 1 and 2. In summary, within-subjects analysis confirms the observation that when participants decide on a particular pair, a higher frequency of risk-averse behavior is observed when that pair is embedded in a set that includes good (end-of-list) pairs.

In addition, Section 4.1 below presents the results of Fischer's exact tests, showing that the observed differences for pairs 6 and 7 between our control Treatment 1 and Treatment 5, as well as the differences between control and Treatment 4 for pair 7 , are statistically significant. Note that treatments 4 and 5 delete the largest numbers of good (end-of-list) pairs.

As we repeatedly noted, good pairs appear at the end of the list. Is the observed effect of deleting good pairs due to their goodness or to their position at the end of the list? We address the issue in the following section.

\begin{tabular}{|c|c|c|c|c|c|c|c|}
\hline & $\begin{array}{l}\text { Treatment } \\
1 \text { (Control) }\end{array}$ & $\begin{array}{c}\text { Treatment } \\
2\end{array}$ & $\begin{array}{c}\text { Treatment } \\
3\end{array}$ & $\begin{array}{c}\text { Treatment } \\
4\end{array}$ & $\begin{array}{l}\text { Treatment } \\
5\end{array}$ & $\begin{array}{c}\text { Average } \\
\text { Treat's } \\
2-5\end{array}$ & $\begin{array}{l}\text { Max Rat } \\
\text { Diff. } \\
\text { Treat's } \\
2-5\end{array}$ \\
\hline Pair 1 & 0.99 & - & - & - & 0.99 & - & - \\
\hline Pair 2 & 0.99 & - & - & 0.99 & 0.98 & - & - \\
\hline Pair 3 & 0.99 & - & 0.96 & 1.00 & 0.98 & - & \\
\hline Pair 4 & 0.97 & 0.99 & 0.94 & 0.99 & 0.97 & 0.97 & 0.05 \\
\hline Pair 5 & 0.89 & 0.91 & 0.86 & 0.87 & 0.80 & 0.86 & 0.14 \\
\hline Pair 6 & 0.71 & 0.76 & 0.69 & 0.63 & 0.58 & 0.66 & 0.28 \\
\hline Pair 7 & 0.34 & 0.36 & 0.30 & 0.19 & 0.18 & 0.26 & 1.00 \\
\hline Pair 8 & 0.11 & 0.11 & 0.05 & 0.06 & - & - & - \\
\hline Pair 9 & 0.03 & 0.01 & 0.01 & - & - & - & - \\
\hline Pair 10 & 0.00 & 0.01 & - & - & - & - & - \\
\hline $\begin{array}{l}\text { Number of } \\
\text { observations }\end{array}$ & 144 & 120 & 94 & 72 & 122 & & \\
\hline
\end{tabular}

Table 3. Rate of safe choices $(S)$ per pair and treatment, Holt and Laury method (in bold the pairs common to all treatments). 
Rate of Safe

Choices

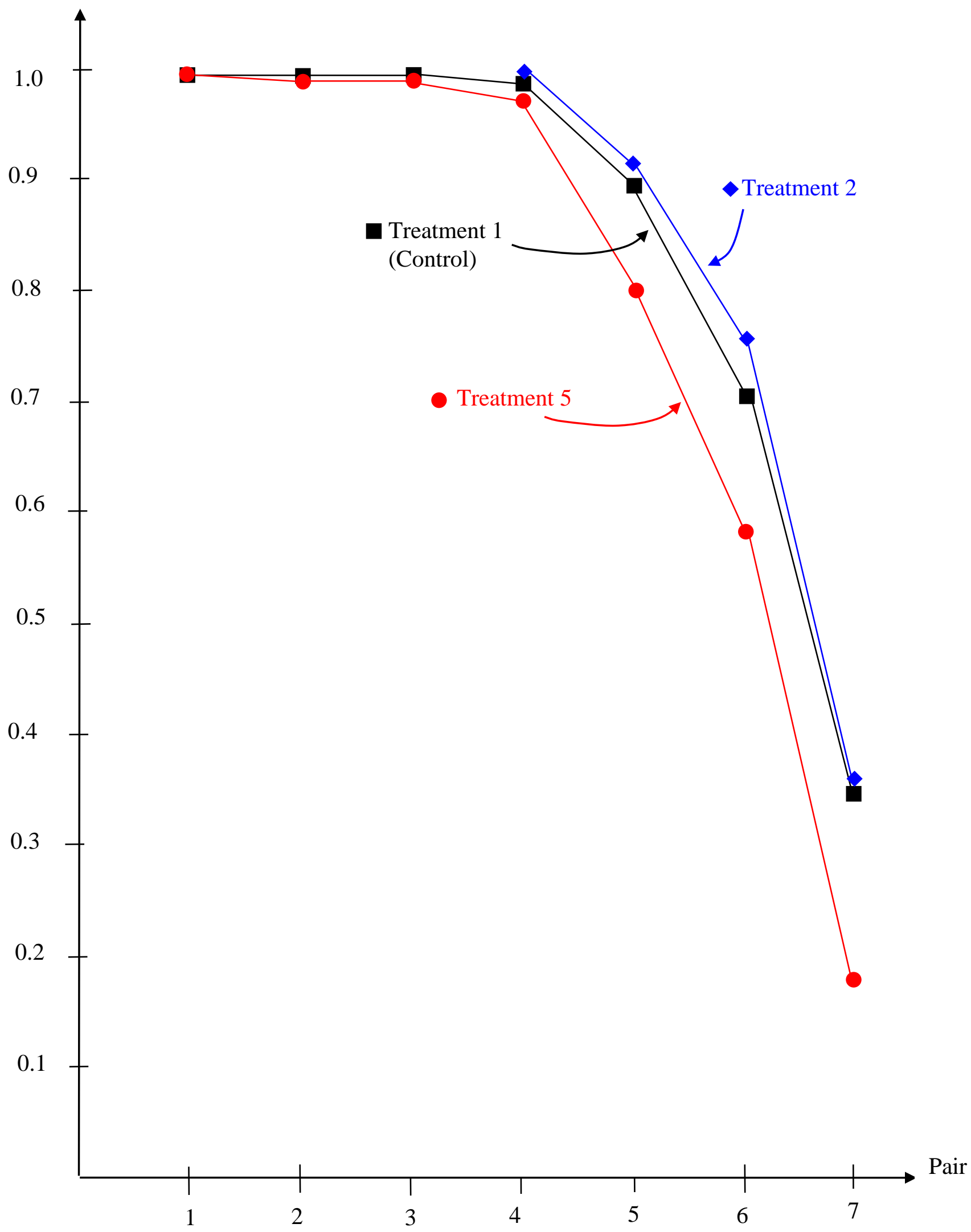

Figure 1. The rate of safe choices $(S)$ in treatments 1,2 and 5 for pairs 1 to 7, Holt and Laury method. 


\subsection{The irrelevance of the position in the list: Inverting the order of the pairs}

The tendency, in some of the shorter lists, to switch earlier from the safe to the risky option brings to mind the phenomenon discussed in Andersen et al. (2006) that, in multiple-price lists, subjects could be inclined to pick a response in the middle of the list, independent of true valuations. An inspection of the results in Table 3 shows that such an attraction for the middle does not appear in our experiment: the middle pair in a list of seven is the fourth one, and the fraction of subjects who choose the safe lottery there goes from 0.36 in Treatment 2 to 0.97 in Treatment 5.

But, as indicated above, the increase in risk aversion as good pairs are removed could conceivably be due to an "end-of-list" effect, since the good pairs are located at the end. One simple way of exploring the issue consists in running the experiment with the order of the pairs inverted, i. e., giving the subjects a list that begins with the better pairs and ends with the worse pairs. If, in the inverted treatments, we observe again that risk aversion is less frequent when removing the better pairs, now located at the beginning of the list, then we will be more confident that it is not the distance to the end of the list that drives the effect.

Accordingly, we have run sessions $A$ to $F$ with the inverted order of pairs. Except for the inversion of the order, the design, procedure and earnings in this experiment are identical to the design, procedure and earnings in the previous one. Again, our subjects were undergraduate students at Universitat Pompeu Fabra who volunteered (totaling 139). The aggregate results are shown in Table 4. One observation is clear from the table, namely that the previously observed effect survives the inversion of the order of lotteries. Moreover, as before, it is more noticeable in pairs 6 and 7. Therefore, we must conclude that the position of the pairs in the list is not what drives the observed bias. 


\begin{tabular}{|c|c|c|c|c|c|c|c|}
\hline & $\begin{array}{l}\text { Treatment } \\
\text { 1i (Control) }\end{array}$ & $\begin{array}{l}\text { Treatment } \\
2 \mathrm{i}\end{array}$ & $\begin{array}{c}\text { Treatment } \\
\text { 3i }\end{array}$ & $\begin{array}{l}\text { Treatment } \\
4 \mathrm{i}\end{array}$ & $\begin{array}{c}\text { Treatment } \\
5 i\end{array}$ & $\begin{array}{l}\text { Average } \\
\text { Treat's } \\
2 i \text { to } 5 i\end{array}$ & $\begin{array}{l}\text { Max Rate } \\
\text { Diff. } \\
\text { Treat's } \\
\text { 2i to } 5 i\end{array}$ \\
\hline Pair 1 & 0.99 & - & - & - & 0.99 & & - \\
\hline Pair 2 & 0.98 & - & - & 0.98 & 0.99 & & - \\
\hline Pair 3 & 0.98 & - & 0.97 & 0.98 & 0.99 & & - \\
\hline Pair 4 & 0.98 & 0.96 & 0.97 & 0.98 & 0.93 & 0.96 & 0.05 \\
\hline Pair 5 & 0.85 & 0.87 & 0.83 & 0.86 & 0.78 & 0.83 & 0.12 \\
\hline Pair 6 & 0.67 & 0.72 & 0.67 & 0.58 & 0.49 & 0.61 & 0.47 \\
\hline Pair 7 & 0.31 & 0.36 & 0.34 & 0.23 & 0.16 & 0.27 & 1.25 \\
\hline Pair 8 & 0.12 & 0.10 & 0.05 & 0.07 & - & - & - \\
\hline Pair 9 & 0.01 & 0.02 & 0.01 & - & - & - & - \\
\hline Pair 10 & 0.00 & 0.00 & - & - & - & - & - \\
\hline $\begin{array}{l}\text { Number of } \\
\text { Observations }\end{array}$ & 171 & 165 & 124 & 108 & 124 & & \\
\hline
\end{tabular}

Table 4. Inverted treatments: Rate of safe choices $(S)$ per pair and treatment in the Holt and Laury method (in bold the pairs common to all treatments). To facilitate the comparison with Table 3, we keep the same ordering of the pairs in both tables. However, one should keep in mind that, in the inverted treatments of Table 4, the order of the lotteries was inverted, so that subjects faced listings of pairs beginning with Pair 10, and ending with Pair 1.

\section{The Abdellaoui et al. method}

\subsection{Our experimental design: Changing list length in Abdellaoui et al.}

Design. We adapt the ADH method by adding one row to their Table 3 in order to facilitate the comparison with the HL procedure. The adapted list appears as our Table $5 .^{7}$ In both the HL and ADH procedures, subjects face a list of "safe" and "risky" pairs, but in ADH the safe alternative is a sure payoff that increases along the list, while the risky one is a 50-50 fixed lottery, that we implement by a coin toss.

We designed five treatments, numbered 1 to 5, and carried them out in five sessions, labeled $A$ to $E$. As in our experiment on the HL method, our Treatment 1 is the control treatment, where subjects face the complete list of Table 5.

\footnotetext{
${ }^{7}$ Here too, the column displaying the expected values was not shown to the experimental subjects, and the columns were labeled $A$ and $B$ instead of $S$ and $R$.
} 


$\begin{array}{lllc}\text { Pair } & \begin{array}{l}\text { Safe } \\ \text { Option }(S)\end{array} & \begin{array}{l}\text { Risky } \\ \text { Option }(R)\end{array} & \begin{array}{l}\text { Expected } \\ \text { payoff diff. }\end{array} \\ 1 & € 5.00 & € 15 \text { if heads, } € 5 \text { if tails } & -5.00 \\ 2 & € 6.10 & € 15 \text { if heads, } € 5 \text { if tails } & -3.90 \\ 3 & € 7.20 & € 15 \text { if heads, } € 5 \text { if tails } & -2.80 \\ 4 & € 8.30 & € 15 \text { if heads, } € 5 \text { if tails } & -1.70 \\ 5 & € 9.45 & € 15 \text { if heads, } € 5 \text { if tails } & -0.55 \\ 6 & € 10.55 & € 15 \text { if heads, } € 5 \text { if tails } & 0.55 \\ 7 & € 11.70 & € 15 \text { if heads, } € 5 \text { if tails } & 1.70 \\ 8 & € 12.80 & € 15 \text { if heads, } € 5 \text { if tails } & 2.80 \\ 9 & € 13.90 & € 15 \text { if heads, } € 5 \text { if tails } & 3.90 \\ 10 & € 15.00 & € 15 \text { if heads, } € 5 \text { if tails } & 5.00\end{array}$

Table 5. Our adaptation of the Abdellaoui et al. design; payoffs in euros.

In treatments 2 to 5 we ran the experiment with lists of seven pairs where some of the better pairs and/or some of the worse pairs of Table 5 (three in total) have been eliminated.

In Treatment 2, each subject faced the seven-pair list obtained by deleting the first three rows (i. e., pairs) of Table 5. In Treatment 3, each subject faced the seven-pair list obtained by deleting rows 1, 2 and 10 of Table 5 . In Treatment 4, each subject faced the seven-pair list obtained by deleting rows 1, 9 and 10 of Table 5. Finally, in Treatment 5 each subject faced the seven-pair list obtained by deleting the last three rows from Table 1 . We were particularly interested in the decisions for pairs 4 to 7 , which are present in all five treatments.

Again, we scrambled the order of our treatments and repeated one of them as a "return to baseline.” In each session, we ran four different treatments in the following orders.

Session $A$, with 21 subjects, implemented treatments 1, 5, 3, 4, 1;

Session $B$, with 21 subjects, treatments 2, 1, 4, 5, 2;

Session $C$ with 20 subjects, treatments 3, 2, 5, 1, 3;

Session $D$, with 21 subjects, treatments 4, 3, 1, 2, 4;

and Session $E$, with 21 subjects, treatments 5, 4, 2, 3, 5 . 
Subjects. Once more, the participants in the experiments were undergraduate students at Universitat Pompeu Fabra (totaling 104), previously registered in a large database for subjects willing to participate in economics experiments. ${ }^{8}$

Procedure. All the sessions were computerized using Z-tree. After the instructions were read and all questions answered, each subject in a session faced a screen with the list corresponding to the first treatment. Each pair in the list contained the two options $S$ and $R$ that, as indicated, in the experiment were called $A$ for the certain quantity in the first column and $B$ for the lottery in the second column. Each list had either 7 or 10 pairs of options depending on the treatment. Once the subjects had chosen either $A$ or $B$ for each pair in a list, the next list for the session appeared on the screen. The session was finished when all five lists in the session had been completed by the subjects.

Earnings. Subjects were told that in order to compute their earnings at the end of the session, each of them individually would randomly pick one of the five lists by drawing a number from an urn containing five numbers. Next, one pair in the list would be randomly picked, again by the subject herself, by drawing one number from an urn containing as many numbers as pairs in the list (7 or 10). Finally, if the subject had chosen Option $A$ for the randomly picked pair, she would be paid the corresponding certain amount, otherwise the lottery would be realized with a coin toss and the subject paid accordingly. The sessions lasted about 30 minutes, and the individual payment procedure was faster than in the HL experiment.

\subsection{Results}

Tables A13 to A17 in Appendix A present the raw experimental data for sessions $A$ to $E$. We can visualize the overall outcomes in Table 6, which displays the rate of safe choices per pair and treatment aggregated over sessions $A$ to $E$ : the format is that of tables 3 and 4 above. ${ }^{9}$

\footnotetext{
${ }^{8}$ Based on the Online Recruitment System for Economic Experiments (ORSEE).

${ }^{9}$ Here we disregard the choices of subjects who switch to $R$ after having already chosen $S$ in a pair, i. e., of the form (...SR...). Again, these compound choices can be shown to be inconsistent in the sense of being first-order stochastically dominated by the compound choice obtained by replacing the $S R$ pair by $R S$.
} 


$\begin{array}{ccccccc} & & & & & \text { Average Max Rat } \\ & & & & & \text { Treat's } & \text { Diff. } \\ \text { Treatment } & \text { Treatment } & \text { Treatment } & \text { Treatment } & \text { Treatment } & 2-5 & \text { Treat's } \\ 1 \text { (Control) } & 2 & 3 & 4 & 5 & & 2-5\end{array}$

$\begin{array}{llllllll}\text { Pair 1 } & 0.00 & - & - & - & 0.00 & - & - \\ \text { Pair 2 } & 0.00 & - & - & 0.00 & 0.00 & - & - \\ \text { Pair 3 } & 0.08 & - & 0.07 & 0.06 & 0.09 & - & - \\ \text { Pair 4 } & \mathbf{0 . 2 9} & \mathbf{0 . 2 4} & \mathbf{0 . 2 3} & \mathbf{0 . 2 9} & \mathbf{0 . 3 7} & 0.28 & 0.51 \\ \text { Pair 5 } & \mathbf{0 . 7 3} & \mathbf{0 . 6 6} & \mathbf{0 . 7 1} & \mathbf{0 . 7 5} & \mathbf{0 . 7 2} & 0.71 & 0.14 \\ \text { Pair 6 } & \mathbf{0 . 9 3} & \mathbf{0 . 9 1} & \mathbf{0 . 9 4} & \mathbf{0 . 9 4} & \mathbf{0 . 9 6} & 0.94 & 0.05 \\ \text { Pair 7 } & \mathbf{1 . 0 0} & \mathbf{0 . 9 8} & \mathbf{0 . 9 8} & \mathbf{0 . 9 7} & \mathbf{0 . 9 7} & 0.98 & 0.03 \\ \text { Pair 8 } & 1.00 & 1.00 & 0.99 & 1.00 & - & - & - \\ \text { Pair 9 } & 1.00 & 1.00 & 1.00 & - & - & - & - \\ \text { Pair 10 } & 1.00 & 1.00 & - & - & - & - & - \\ & & & & & & & \end{array}$

Table 6. Rate of safe choices (S) per pair and treatment for the Abdellaoui et al. method (in bold, the pairs common to all treatments).

The inspection of Table 6 shows that the pairs more affected by the deletions are pairs 4 and 5. By comparing the first two columns of Table 6 (Treatment 2 vs. control), we observe that the deletion of the first three (worse) pairs slightly decreases the rate of safe choices. Treatment 5 , which deletes the three last (best) pairs, shows no consistent pattern of differences from control. In any event, as we will see in Section 4.1 below, Fischer's exact test indicates that the differences are not statistically significant. In all fairness, it should be noted that the number of subjects is lower in our test of the ADH method (104) than in that of the HL method (145), and that statistical significance is easier to obtain with the larger number.

\section{Discussion}

\subsection{Comparing Holt and Laury with Abdellaoui et al.}

The main lesson of our experiments is that the HL and ADH methods respond quite differently to the deletions of pairs. We have performed Fischer's exact test for both methods, focusing on pairs 
6 and 7 in HL, and pairs 4 and 5 in ADH, the pairs that show larger responses to the deletions. The results are displayed in Table 7.

\begin{tabular}{|l|l|l|l|l|}
\cline { 2 - 5 } \multicolumn{1}{c|}{} & \multicolumn{2}{c|}{ Holt and Laury method } & \multicolumn{2}{l|}{ Abdellaoui et al. method } \\
\cline { 2 - 5 } \multicolumn{1}{c|}{} & Pair 6 & Pair 7 & Pair 4 & Pair 5 \\
\hline $\begin{array}{l}\text { Treatment 1 } \\
\text { vs. Treatment 2 }\end{array}$ & 0.40 & 0.79 & 0.43 & 0.36 \\
\hline $\begin{array}{l}\text { Treatment 1 } \\
\text { vs. Treatment 3 }\end{array}$ & 0.88 & 0.57 & 0.27 & 0.76 \\
\hline $\begin{array}{l}\text { Treatment 1 } \\
\text { vs. Treatment 4 }\end{array}$ & 0.28 & $\mathbf{0 . 0 3 9}$ & 1.00 & 0.75 \\
\hline $\begin{array}{l}\text { Treatment 1 } \\
\text { vs. Treatment 5 }\end{array}$ & $\mathbf{0 . 0 3 9}$ & $\mathbf{0 . 0 0 3 5}$ & 0.30 & 1.00 \\
\hline
\end{tabular}

Table 7. Double-tail Fischer’s exact test $p$-values. Significant results in bold.

We observe that the differences between treatments and control are not significant for the ADH method. But in the case of the HL method, and confirming our observations in Section 2.4 above, we obtain significant differences for Treatment 4 and Pair 7, and for Treatment 5 and pairs 6 and 7. Recall that in Treatment 4 (resp. 5) we delete the two (resp. three) best pairs.

The inspection of the last two columns of tables 3, 4 and 6 provides an informal confirmation of the asymmetry. Averaging over treatments 2 to 5 yields numbers that are relatively close to control in ADH, whereas they are markedly different for pairs 6 and 7 in the HL method. And the maximal-rate differences for treatments 2 to 5 are typically larger in the HL method than in ADH's, indicating more dispersion.

That the HL method suffers from an embedding bias, while the ADH method does not, cannot be attributed to HL testing for a more restrictive version of risk aversion than ADH, as it could be the case if the "risky" prospects in the HL method were a mean-preserving spread of the "safe” prospects.

Observe also that the HL procedure is more complex than that of ADH, and hence it generates nosier responses. Indeed, the fraction of subjects who display inconsistent behavior in the abovementioned sense of switching back and forth, in a first-order stochastically-dominated way, while small in absolute terms, is larger in the HL method than in ADH's. Despite the added noise, however, we do find a clear, systematic embedding bias in the HL method. 


\subsection{Violations of the independence axiom}

Our result that, in the HL method, the deletion of some specific pairs favors an earlier switch to the riskier option shows that the choice in a given pair is not independent from the list where it is embedded. Hence, a subject displaying such behavior cannot be maximizing preferences that satisfy the independence axiom and, therefore, the expected utility hypothesis (see, e. g., MasColell et al., 1995). Consequently, any formalization of this behavior must discard the independence axiom. It follows from our experimental results that the repeated reliance on vNM utility functions by Holt and Laury (2002) and subsequent users of the HL method is not well grounded, despite the awareness previously evidenced by Holt (1986).

In addition to violations of the independence axiom in decisions under uncertainty, the literature presents other instances of the dependence of the value, or category, assigned to a particular item on the set of items in which it is embedded. In the medical literature, Robinson et al. (2001) observe how respondents' rankings of descriptions of road injuries depend on the set of descriptions in which there are included. In psychology, Parducci and Weddell (1986) define a "range-frequency effect” where the category assigned to the size of a square (e. g., large, or small) depends both on the number of allowable categories and on the support and the frequency of the distribution of sizes in the list presented to the subject. Stewart et al. (2005) ascertain the importance of the intensity difference between a stimulus and the previous one in the sequence. In marketing research, Huber et al. (1982) study consumers' choices when confronted with a set of products each of which is favored in a different dimension (size, quality, color, price), and observe that introducing a new product that is dominated in all dimensions by one of the existing products results in the latter product being hugely favored by consumers. This is an instance of how asymmetric dominance may affect choices, as analyzed by Amaldoss et al. (2008).

\subsection{Increasing risk aversion and generalized expected utility}

Machina $(1982,1983)$ tackled the more common observed violations of the independence axiom by proposing a generalized expected utility model characterized by the smoothness of the utility function defined on the space of probability distributions, so that a local vNM-type utility function can be defined at each probability distribution. He showed that the most common violations of the independence axiom (the effects named common consequence, common ratio, oversensitivity to changes in small- probability outlying events, and utility evaluation) were implied by an elegant 
condition, which he called Hypothesis II, by which the local vNM-type utility function of a better probability distribution (in the sense of first-order stochastic dominance) is more concave, implying more risk aversion, than the one corresponding to a worse probability distribution. ${ }^{10}$ Hence, under Hypothesis II the decision maker displays a higher degree of risk aversion in the neighborhood of a better probability distribution than in the neighborhood of a worse one. Accordingly, Machina's Hypothesis II suggests a justification for our experimental result that, in the HL method, adding better options favors risk aversion. ${ }^{11}$

But we only observe such an increase in risk aversion for the HL method, and not in the ADH method, while in either case we delete the better pairs when we move from Treatment 1 (full-ten pair list) to Treatment 5. One possible explanation is that, while in both methods the deletion of the last three pairs yields a worse set, the worsening is stronger in the HL method than in the ADH method.

The analysis presented in Appendix B below points in that direction. In either method, the set of compound choices available under the whole list is "better" than the one under the reduced list in the following sense: each compound choice in the "better" set first-order stochastically dominates (FOSD) at least one compound choice in the "worse” set. In the HL method, many of the compound choices of the "better" set FOSD three, four, five and even six compound choices of the "worse" set, whereas in the ADH method they only FOSD one or two of the "worse" set. It is also the case that, in the HL method, such first-order stochastic domination yields a larger increase in expected money values than in the ADH method.

\subsection{Does risk taking increase when removing pairs where the risky option is more popular?}

As noted, the ADH method does not show a statistically significant dependence of the frequency of safe choices on the deletions. Yet some deviations from control do occur, particularly in Treatment 2, in which the rate of safe choices is smaller than in control. Now, in Treatment 2, it is the worse choice pairs that have been deleted: this goes in the opposite direction to the bias

\footnotetext{
${ }^{10}$ For the case of three outcomes, Hypothesis II implies that the indifference curves "fan out" in the MarschakMachina triangle.

${ }^{11}$ By the same token, Machina's Hypothesis II may apply to a similar finding by Bateman et al. (2007): in the process of analyzing the preference reversal phenomenon, they find that the certainty equivalent of a given lottery (say, Lottery I or Lottery $\mathrm{J}$ in their Table 1) is lower when included in a list of better lotteries (Set 1 ibid.) than when included in a set of worse lotteries (Set 2 ibid.).
} 
displayed by the HL method, where it is the deletion of the better prospects that decreases risk aversion.

Notice, however, that in the HL method most subjects, when confronted with the better pairs, choose the risky option, whereas in the ADH method most subjects choose the risky alternative in the worse pairs. Perhaps, then, what drives the changes in risk aversion is the deletion of pairs where most subjects choose the risky alternative, rather than the deletion of better or worse pairs.

Table 8 offers a comparative summary of the features associated with decreased risk aversion in the HL and ADH methods.

\begin{tabular}{|l|l|l|}
\hline $\begin{array}{l}\text { Holt and Laury } \\
\text { Method }\end{array}$ & $\begin{array}{l}\text { Removal of good pairs } \\
\text { = Removal of pairs where most } \\
\text { subjects choose the risky option }\end{array}$ & $\begin{array}{l}\text { fewer safe choices } \\
\text { = less risk aversion } \\
\text { (statistically significant) }\end{array}$ \\
\hline $\begin{array}{l}\text { Abdellaoui et al. } \\
\text { Method }\end{array}$ & $\begin{array}{l}\text { Removal of bad pairs } \\
\text { = Removal of pairs where most } \\
\text { subjects choose the risky option }\end{array}$ & $\begin{array}{l}\rightarrow \text { fewer safe choices } \\
\text { = less risk aversion } \\
\text { (not statistically significant) }\end{array}$ \\
\hline
\end{tabular}

Table 8. Comparison of the features associated with decreased risk aversion

We have pondered three explanations for the phenomenon of decreased risk aversion clearly observed in the HL method, namely the deletion of (a) better pairs, (b) end-of-list pairs, and (c) pairs where the risky option is more popular. Our analysis in Section 2.4 above definitely rules (b) out. If we attached any importance to the statistically not significant changes observed in our test of the $\mathrm{ADH}$ method, then we would have to rule (a) out, leaving only (c). But at this point, in the absence of further research, it would be reckless to bet on any of these explanations.

Finally, recall that the HL method deals with a list of varying probabilities while the ADH method only makes use of one and the same probability in its list. One could conjecture that an embedding bias may appear whenever a different embedding frame shifts the individual probability weighting functions and, therefore, the degree of observed risk aversion. More so since our own method for eliciting risk preferences (Bosch-Domènech and Silvestre, 1999, 2006a, $b, c$, 2010) -which like ADH's uses only one and the same probability in its lotteries- appears also to be immune to the embedding bias. 


\section{Conclusions}

The paper tests the robustness of experimental procedures aimed at measuring risk aversion, where subjects face a list of pairs of alternative prospects. More specifically, we examine whether the removal of some items of the list affects the outcomes, focusing on the widely used Holt and Laury (2002) method and on the more recent one by Abdellaoui et al. (2011). Either method uses a list of pairs: we conduct experiments where some pairs are removed from the list. We ask: are decisions on a particular pair influenced by the presence or absence of other pairs in the list?

We experimentally discover a systematic bias, that we call embedding bias, in the Holt and Laury method: as some specific pairs are removed, risk aversion becomes less frequent and the ranking of individuals by risk aversion is not preserved. No statistically significant bias appears, on the contrary, in our test of the Abdellaoui et al. method, which, unlike Holt and Laury's, does not use a list with varying probabilities.

The pairs whose deletion induces the reduction of risk aversion in the Holt and Laury method are the better pairs and, at the same time, the pairs where most subjects choose the risky option, and also the last ones in their list. By repeating our experiment with the order of pairs inverted, we find that the position of the pairs in the list is irrelevant.

But it would be premature to explain the phenomenon in terms of the removal of good pairs: even though the results for our test of Abdellaoui et al. are not statistically significant, they point away from this explanation. It remains to be seen whether it is the use of a list of different probabilities which makes the Holt and Laury method vulnerable to the embedding bias, a possibility that gains some weight considering that our own method for measuring risk aversion which like Abdellaoui et al. involves only one fixed probability- is also immune to the embedding bias.

In conclusion, our experimental results uncover a new bias that puts into question the robustness of some procedures for measuring individual behavior based on lists of alternative prospects and, in particular, provide a new call for caution when using the Holt and Laury method to estimate individual risk aversion and to rank individuals by their risk aversion. It also suggests that better alternatives may already exist. 


\section{APPENDIX A}

\begin{tabular}{|c|c|c|c|c|}
\hline 5 & eatment 3 & tment 2 & Treatment 1 & Trea \\
\hline & 00SSSSS/RR0 & & RRRR & \\
\hline 00 & RR0 & 000SSSS/RRR & S/RRR & SS \\
\hline SSSS/RR000 & 00SSSS/RRR0 & 000SSSS/RRR & SSSSSSS/RRR & SSSSSS/R000 \\
\hline S/R000 & 00SSSS/RRR0 & 000SSSS/RRR & SSSSS/RRR & SSSSSS/R000 \\
\hline SSSSS000 & OSSSSSS/R0 & 000Ss & $\mathrm{SS} / \mathrm{R}$ & 500 \\
\hline 000 & SSSS/RRR0 & RRR & /RRR & 00 \\
\hline SSSSSS000 & 00SSSSS/RR0 & 00SSSS/RRR & SSSSSSS/RRR & SSSSS/ \\
\hline SSSSS/RR000 & OSSS/RRRR0 & RRRR & RRRRR & SSSS/R \\
\hline 0 & RR0 & SS/RR & B/RRR & SSSS \\
\hline SSSSS/R000 & 00SSSS/RRR0 & 000SSSSS/RR & SSSSSSS/RRR & SSSSS \\
\hline 5000 & /RR0 & S/RR & RRRR & $\mathrm{S} / \mathrm{RI}$ \\
\hline SSSS & 20 & $\mathrm{RR}$ & RR & 000 \\
\hline 00 & R0 & RRR & $\mathrm{RR}$ & 00 \\
\hline SSSS & R0 & /RRR & RR & 2000 \\
\hline$S S S / R$ & $00 \mathrm{SS} / \mathrm{R}$ & 000S/R & SSSSS & SSSS \\
\hline $\mathrm{SSSS} / \mathrm{H}$ & RR0 & RRR & RRR & R000 \\
\hline SS & RR0 & $\mathrm{R} / \mathrm{S}$ & $\mathrm{S} / \mathrm{RR}$ & $\mathrm{S} / \mathrm{F}$ \\
\hline SSSS & Ro & $\mathrm{RR}$ & RRR & 000 \\
\hline SSSSSS/ & RR0 & 000SS & RRR & 2000 \\
\hline SSSSS & S/RR0 & S/RRR & SS/RR & SSSSSSS000 \\
\hline 2000 & RRR0 & RRRR & SSSSS/RRRRR & SSSS/RRR00 \\
\hline SSSSSS/R000 & 00SSS/RRRR0 & /RRRR & SSSSS/RRRRR & SSSSSS/R000 \\
\hline SSSSSS/R000 & SS/RRR0 & SS/RRR & SSSSSS/RRRR & SSSSSSS000 \\
\hline SSSSS/RR000 & S/RRR0 & RRRRR & SSSSS/RRRRR & SSSSS/RR00 \\
\hline SSSSS/RR000 & 00SSS/RRRR0 & 000SS/RRRRR & SSSS/RRRRRR & SSSSS/RR000 \\
\hline SSS/RR & 00SS/RRRRR0 & 000S/RRRRRR & SSSS/RRRRRR & SSSSSSS000 \\
\hline & & & & \\
\hline SSSS/RR000 & 00SSSSS/RR0 & 00SSS/RRRR & SSSSSS/RRRR & SSSSSS/R00 \\
\hline
\end{tabular}

Table A1. Choices of subjects (28) in Session A for the Holt and Laury method (recall that zeroes mark the deletion of pairs). 


$\begin{array}{lllll}\text { Treatment } 2 & \text { Treatment } 4 & \text { Treatment } 5 & \text { Treatment } 1 & \text { Treatment } 2 \\ \text { 000SSS/RRRR } & \text { 0SSSS/RRR00 } & \text { SSSSS/RR000 } & \text { SSSS/RRRRRR } & \text { 000SS/RRRRR } \\ \text { 000SSS/RRRR } & \text { 0SSSSS/RR00 } & \text { SSSSSS/R000 } & \text { SSSSSS/RRRR } & \text { 000SSS/RRRR } \\ \text { 000SSS/RRRR } & \text { 0SSSSS/RR00 } & \text { SSSSSS/R000 } & \text { SSSSSS/RRRR } & \text { 000SSS/RRRR } \\ \text { 000SSS/RRRR } & \text { 0SSSS/RRR00 } & \text { SSSSS/RR000 } & \text { SSSSS/RRRR } & \text { 000SSS/RRRR } \\ \text { 000SSS/RRRR } & \text { 0SSSSS/RR00 } & \text { SSSSSS/R000 } & \text { SSSSSS/RRRR } & \text { 000SSS/RRRR } \\ \text { 000SSS/RRRR } & \text { 0SSSSS/RR00 } & \text { SSSSSS/R000 } & \text { SSSSSS/RRRR } & \text { 000SSS/RRRR } \\ \text { 000SSS/RRRR } & \text { 0SSSSS/RR00 } & \text { SSSSSS/R000 } & \text { SSSSSS/RRRR } & \text { 000SSS/RRRR } \\ \text { 000SSS/RRRR } & \text { 0SSSS/RRR00 } & \text { SSSSS/RR000 } & \text { SSSSSS/RRRR } & \text { 000SSS/RRRR } \\ \text { 000SSS/RRRR } & \text { 0SSSSS/RR00 } & \text { SSSSS/RR000 } & \text { SSSSSS/RRRR } & \text { 000SSS/RRRR } \\ \text { 000SSSSS/RR } & \text { 0SSSSS/RR00 } & \text { SSSSSS/R000 } & \text { SSSSSSS/RRR } & \text { 000SSSSS/RR } \\ \text { 000SS/RRRRR } & \text { 0SSSS/RRR00 } & \text { SSS/RRRR000 } & \text { SSSS/RRRRRR } & \text { 000S/RRRRRR } \\ \text { 000SSS/RRRR } & \text { 0SSSSS/RR00 } & \text { SSSSSS/R000 } & \text { SSSSS/RRRRR } & \text { 000SSS/RRRR } \\ \text { 000S/RRRRRR } & \text { 0SSS/RRRR00 } & \text { SSSS/RRR000 } & \text { SSSSSS/RRRR } & \text { 000S/RRRRRR } \\ \text { 000SS/RRRRR } & \text { 0SSSS/RRR00 } & \text { SSSSS/RR000 } & \text { SSSSS/RRRRR } & \text { 000SS/RRRRR } \\ \text { 000SS/RRRRR } & \text { 0SSS/RRRR00 } & \text { SSSSS/RR000 } & \text { SSSSS/RRRRR } & \text { 000SSS/RRRR } \\ \text { 000SSS/RRRR } & \text { 0SSSSS/RR00 } & \text { SSSSSS/R000 } & \text { SSSSSS/RRRR } & \text { 000SSS/RRRR } \\ \text { 000SS/R/S/RRR } & \text { 0SSSS/RRR00 } & \text { SS/R/S/RRR000 } & \text { SSSSS/RRRRR } & \text { 000SSS/RRRR } \\ \text { 000SSS/RRR/S } & \text { 0SSS/RRR/S00 } & \text { SSS/RRR/S000 } & \text { SSSSSSSSSS } & \text { 000RRR/SSSS } \\ \text { 000SSSS/RRR } & \text { 0SSSSS/RR00 } & \text { SSSSSS/R000 } & \text { SSSSSSS/RRR } & \text { 000SSSS/RRR } \\ \text { 000SSSS/RRR } & \text { 0SSSSS/RR00 } & \text { SSSSSSS000 } & \text { SSSSSSS/RRR } & \text { 000SSSS/RRR } \\ \text { 000S/RRRRRR } & \text { 0SSS/RR/S/R00 } & \text { SS/R/S/RRR000 } & \text { SSS/RRR/S/RRR } & \text { 000SSS/RRRR } \\ \text { 000SSSS/RRR } & \text { 0SSSSS/RR00 } & \text { SSSSSS/R000 } & \text { SSSSSSS/RRR } & \text { 000SSSS/RRR } \\ \text { 000SS/RRRRR } & \text { 0SSSS/RRR00 } & \text { SSSSSS/R000 } & \text { SSSSS/RRRRR } & \text { 000SS/RRRRR } \\ \text { 000SS/RRRRR } & \text { 0SSSS/RRR00 } & \text { SSSSS/RR000 } & \text { SSSSSS/RRRR } & \text { 000SS/RRRRR }\end{array}$

Table A2. Choices of subjects (24) in Session B for the Holt and Laury method. 


\begin{tabular}{|c|c|c|c|c|}
\hline 1 & 5 & t 3 & 2 & - \\
\hline & & R0 & $\mathrm{RR}$ & $\mathrm{RP}$ \\
\hline RRR & SSSS/1 & 0SSSSS/RR0 & RRRR & RRRR \\
\hline RR & 00 & R0 & 000S & RR \\
\hline SSSSS/RRR & SSSSSSS000 & 00SSSSS/RR0 & 000SSSS/RRR & SSSSSS \\
\hline RRR & SSS & 00Ss & $000 \mathrm{~S}$ & RRR \\
\hline RR & SSS & 00Ss & 000 & RR \\
\hline $\mathrm{R}$ & 0 & R0 & 000 & RR \\
\hline $\mathrm{R}$ & SS & R0 & $\mathrm{RR}$ & RRR \\
\hline S/RRRR & SSSSS & & $000 s$ & RRRR \\
\hline $\mathrm{R}$ & SSS & $00 s$ & $\mathrm{RR}$ & $\mathrm{RR}$ \\
\hline RR & SSS & $00 \mathrm{~S}$ & 000 & SS \\
\hline RR & SSS & $00 \mathrm{~S}$ & RR & RRR \\
\hline RR & & & & RRR \\
\hline RRR & SSS & R0 & RR & RRR \\
\hline SSSS & SSSS & RRR0 & /RRR & $\mathrm{S} / \mathrm{RRR}$ \\
\hline SSSS/RRRRR & SSSSS/RR000 & 00SSSS/RRR0 & 000SSSS/RRR & SSSSSS/RRRR \\
\hline RRR & SSSS & 00S & RRR & RRR \\
\hline RRRR & SSSS & 00Ss & & SSS \\
\hline SSSSSS/RRRR & SSSS/RRR000 & 00SSS/RRRR0 & 000SS/RRRRR & SSSSSS/RRRR \\
\hline SSSSSSS/RRR & SSSSSS/R000 & 00SSSS/RRR0 & 000SSS/RRRR & SSSSSSS/RRR \\
\hline SSS/RRRRRR & SSSSSSS000 & 00SSSS/RRR0 & 000SSSS/RRR & SSSSSSS/RRR \\
\hline
\end{tabular}

Table A3. Choices of subjects (21) in Session $C$ for the Holt and Laury method. 


$\begin{array}{lllll}\text { Treatment } 3 & \text { Treatment } 5 & \text { Treatment } 2 & \text { Treatment } 1 & \text { Treatment } 3 \\ \text { 00SSS/RRRR0 } & \text { SSSS/RRR000 } & \text { 000SSS/RRRR } & \text { SSSSS/RRRRR } & \text { 00SSS/RRRR0 } \\ \text { 00SSS/RRRR0 } & \text { SSSS/RRR000 } & \text { 000SS/RRRRR } & \text { SSSS/RRRRR } & \text { 00SS/RRRRR0 } \\ \text { 00SSS/RRRR0 } & \text { SSSSS/RR000 } & \text { 000SSS/RRRR } & \text { SSSSSS/RRRR } & \text { 00SSSS/RRR0 } \\ \text { 00SSSSS/RR0 } & \text { SSSSSSS000 } & \text { 000SSSS/RRR } & \text { SSSSSSS/RRR } & \text { 00SSSSS/RR0 } \\ \text { 00SS/RRRRR0 } & \text { SSS/RRRR000 } & \text { 000RRRRRRR } & \text { SSS/R/S/RRRR } & \text { 00SS/RRRRR0 } \\ \text { 00SSS/RRRR0 } & \text { SSSSS/RR000 } & \text { 000SSS/RRRR } & \text { SSSS/RRRRRR } & \text { 00SSS/RRRR0 } \\ \text { 00SSSS/RRR0 } & \text { SSSSS/RR000 } & \text { 000SSS/RRRR } & \text { SSSSSS/RRRR } & \text { 00SSSS/RRR0 } \\ \text { 00SSS/RRRR0 } & \text { SSSS/RRR000 } & \text { 000S/RRRRRR } & \text { SSSSSS/RRRR } & \text { 00SSSS/RRR0 } \\ \text { 00SSS/RRRR0 } & \text { SSSS/RRR000 } & \text { 000SS/RRRRR } & \text { SSSSS/RRRRR } & \text { 00SSS/RRRR0 } \\ \text { 00SS/RRRRR0 } & \text { SSSS/RRR000 } & \text { 000SS/RRRRR } & \text { SSSSS/RRRRR } & \text { 00SS/RRRRR0 } \\ \text { 00SSSSS/RR0 } & \text { SSSSSS/R000 } & \text { 000SSSS/RRR } & \text { SSSSSSS/RRR } & \text { 00SSSSS/RR0 } \\ \text { 00SSSSS/RR0 } & \text { SSSSSS/R000 } & \text { 000SSS/RRRR } & \text { SSSSSS/RRRR } & \text { 00SSSS/RRR0 } \\ \text { 00SSSSS/RR0 } & \text { RR/S/R/SSS000 } & \text { 000RR/S/R/SSS } & \text { RR/S/R/SSSS/RR } & \text { 00RR/S/R/SSS0 } \\ \text { 00SS/R/S/RRR1 } & \text { SSSSSSS000 } & \text { 000SSSSS/RR } & \text { SSSSSSS/RRR } & \text { 00SSSSS/RR0 } \\ \text { 00SSSSS/RR0 } & \text { SSSSSS/R000 } & \text { 000SSS/RRRR } & \text { SSSSSSS/RRR } & \text { 00SSSSS/RR0 } \\ \text { 00SSSSS/RR0 } & \text { SSSSSSS000 } & \text { 000SSSS/RRR } & \text { SSSSSSS/RRR } & \text { 00SSSSS/RR0 } \\ \text { 00SS/RRRRR0 } & \text { SSS/RRRR000 } & \text { 000RRRRRRR } & \text { SSSSS/RRRRR } & \text { 00SSS/RRRR0 } \\ \text { 00SSSSS/RR0 } & \text { SSSSSS/R000 } & \text { 000SSS/RRRR } & \text { SSSSSS/RRRR } & \text { 00SSSS/RRR0 } \\ \text { 00SSSS/R/S/RC } & \text { SSSSS/RR000 } & \text { 000SSS/RRRR } & \text { SSSSSSSS/RR } & \text { 00SSSSS/RR0 } \\ \text { 00RRRRRRR0 } & \text { SSSSSS/R000 } & \text { 000SSS/RRRR } & \text { SSSSSS/RRRR } & \text { 00SSSS/RRR0 } \\ \text { 00RRRRRRR0 } & \text { SSSS/RRR000 } & \text { 000RRRRRRR } & \text { SSSS/RRRRRR } & \text { 00SS/RRRRR0 } \\ \text { 00SSSSS/RR0 } & \text { SSSS/RRR000 } & \text { 000SSSS/RRR } & \text { SSSSSSS/RRR } & \text { 00SSSSS/RR0 } \\ \text { 00SS/RRRRR0 } & \text { SSSS/RRR000 } & \text { 000SSS/RRRR } & \text { SSSS/RRRRRR } & \text { 00SSS/RRRR0 } \\ \text { 00SSSSSSS0 } & \text { SSSSSSS000 } & \text { 000SSSSSS/R } & \text { SSSSSSSSS/R } & \text { 00SSSSSSS0 }\end{array}$

Table A4. Choices of subjects (24) in Session $D$ for the Holt and Laury method. 


$\begin{array}{lllll}\text { Treatment } 4 & \text { Treatment } 1 & \text { Treatment } 2 & \text { Treatment } 3 & \text { Treatment } 4 \\ \text { 0SSSSS/RR00 } & \text { SSSSSS/RRRR } & \text { 000SSS/RRRR } & \text { 00SSSS/RRR0 } & \text { 0SSSSS/RR00 } \\ \text { 0SSSS/RSR00 } & \text { SSSSSSS/RRR } & \text { 000SSSS/RRR } & \text { 00SSSSS/RR0 } & \text { 0SSSSS/RR00 } \\ \text { 0SS/RRRRR00 } & \text { SSS/RRRRRRR } & \text { 000RRRRRRR } & \text { 00S/RRRRRR0 } & \text { 0SS/RRRRR00 } \\ \text { 0SSSSS/RR00 } & \text { SSSSSS/RRRR } & \text { 000SSS/RRRR } & \text { 00SSSS/RRR0 } & \text { 0SSSSS/RR00 } \\ \text { 0SSS/RSRR00 } & \text { SSSSS/RRRRR } & \text { 000SSSSS/RR } & \text { 00SSSSS/RR0 } & \text { 0SSSSS/RR00 } \\ \text { 0SSSSSS/R00 } & \text { SSSSSSSS/RR } & \text { 000SSSSS/RR } & \text { 00SSSSSS/R0 } & \text { 0SSSSSS/R00 } \\ \text { 0SSSS/RRR00 } & \text { SSSSSS/RRRR } & \text { 000SSSS/RRR } & \text { 00SSSS/RRR0 } & \text { 0SSSS/RRR00 } \\ \text { 0SSSS/RRR00 } & \text { SSSS/RRRRRR } & \text { 000SS/RRRRR } & \text { 00SSS/RRRR0 } & \text { 0SSSS/RRR00 } \\ \text { 0SSS/RRRR00 } & \text { SSSSS/RRRRR } & \text { 000SSS/RRRR } & \text { 00SSS/RRRR0 } & \text { 0SSSS/RRR00 } \\ \text { 0SSSSS/RR00 } & \text { SSSSSS/RRRR } & \text { 000SSS/RRRR } & \text { 00SSSS/RRR0 } & \text { 0SSSS/RRR00 } \\ \text { 0SSSSS/RR00 } & \text { SSSSSS/RRRR } & \text { 000SSS/RRRR } & \text { 00SSSS/RRR0 } & \text { 0SSSSS/RR00 } \\ \text { 0SSS/RRRR00 } & \text { SSSS/RSRRRR } & \text { 000S/RRRRRR } & \text { 00RRRRRR0 } & \text { 0SSS/RRRR00 } \\ \text { 0SSSSS/RR00 } & \text { SSSSSS/RRRR } & \text { 000SSS/RRRR } & \text { 00SSSS/RRR0 } & \text { 0SSSSS/RR00 } \\ \text { 0SSSSS/RR00 } & \text { SSSSSSS/RRR } & \text { 000SSSS/RRR } & \text { 00SSSSS/RR0 } & \text { 0SSSSS/RR00 } \\ \text { 0RSRRRRR00 } & \text { SSRSRRSRR } & \text { 000SSS/RRRR } & \text { 00SSSS/RRR0 } & \text { 0SSSSS/RR00 } \\ \text { 0SSS/RRRR00 } & \text { SSSSSSS/RRR } & \text { 000SSS/RRRR } & \text { 00SSSSS/RR0 } & \text { 0SSSSSS/R00 } \\ \text { 0SSS/RRRR00 } & \text { SSSSS/RRRRR } & \text { 000SS/RRRRR } & \text { 00SSSSS/RR0 } & \text { 0SSSS/RRR00 } \\ \text { 0SSSS/RRR00 } & \text { SSSSS/RRRRR } & \text { 000SS/RRRRR } & \text { 00SSS/RRRR0 } & \text { 0SSSSS/RR00 } \\ \text { 0SSSS/RRR00 } & \text { SSSSSS/RRRR } & \text { 000SSS/RRRR } & \text { 00SSSS/RRR0 } & \text { 0SSSSS/RR00 } \\ \text { 0SSSSS/RS00 } & \text { SSSSSS/RRRR } & \text { 000SSS/RRRR } & \text { 00SSSS/RRR0 } & \text { 0SSSSSS/R00 } \\ \text { 0SSSSS/RR00 } & \text { SSSSSS/RRRR } & \text { 000SSS/RRRR } & \text { 00SSSSS/RR0 } & \text { 0SSSSSS/R00 } \\ \text { 0SSSSS/RR00 } & \text { SSSSSS/RRRR } & \text { 000SS/RRRRR } & \text { 00SSSS/RRR0 } & \text { 0SSSSS/RR00 }\end{array}$

Table A5. Choices of subjects (22) in Session $E$ for the Holt and Laury method. 


\begin{tabular}{|c|c|c|c|c|}
\hline Treatment 1 & Treatment 4 & Treatment 2 & Treatment 5 & Treatment 4 \\
\hline SSSSSSSS/RR & 0SSSSSS/R00 & 000SSSS/RRR & SSSSSSS000 & 0SSSSSS/R00 \\
\hline SSSSSS/RRRR & 0SSS/RRRR00 & 000SSS/RRRR & SSSS/RRR000 & 0SSS/RRRR00 \\
\hline SSSSSS/RRRR & 0SSSSS/RR00 & 000SSS/RRRR & SSSSSS/R000 & 0SSSSS/RR00 \\
\hline SSSSSS/RRRR & 0SSSSS/RR00 & 000SSS/RRRR & SSSSSS/R000 & 0SSSSS/RR00 \\
\hline SSSSSS/RRRR & 0SSSSSS/R00 & 000SSSS/RRR & SSSSSSS000 & 0SSSSSS/R00 \\
\hline SSSSSSS/RRR & 0SSSSS/RR00 & 000SS/RRRRR & SSSSSS/R000 & 0SSSSS/RR00 \\
\hline SSSSS/RRRRR & 0SSSSS/RR00 & 000SSS/RRRR & SSSSSS/R000 & 0SSSSS/RR00 \\
\hline SSSSSS/RRRR & 0SSSS/RRR00 & 000SS/RRRRR & SSSSSS/R000 & 0SSSSS/RR00 \\
\hline SSSSSSS/RRR & 0SSSSSS/R00 & 000SSSS/RRR & SSSSSS/R000 & 0SSSSSS/R00 \\
\hline SSSSS/RRRRR & 0SSSS/RRR00 & 000SS/RRRRR & SSSSS/RR000 & 0SSSS/RRR00 \\
\hline SSS/RRRRRRR & 0SSS/RRRR00 & 000S/RRRRRR & SSSS/RRR000 & 0SSS/RRRR00 \\
\hline SSSSSSS/RRR & 0SSSSSS/R00 & 000SSSS/RRR & SSSSSS/R000 & 0SSSSS/RR00 \\
\hline SSSSSSS/RRR & 0SSSSSS/R00 & 000SSSS/RRR & SSSSSS/R000 & 0SSSSS/RR00 \\
\hline SSSSSS/RRRR & 0SSSSS/RR00 & 000SSS/RRRR & SSSSSS/R000 & 0SSSSS/RR00 \\
\hline SSSSSSS/RRR & 0SSSSS/RR00 & 000SSS/RRRR & SSSSSS/R000 & 0SSSSS/RR00 \\
\hline SSSSSSSS/RR & 0SSSSSS/R00 & 000SSSSS/RR & SSSSSSS000 & 0SSSSSSS00 \\
\hline SSSSS/RRRRR & 0SSSS/RRR00 & 000SS/RRRRR & SSSSS/RR000 & 0SSSSS/RR00 \\
\hline SSSSSS/RRRR & 0SSSS/RRR00 & 000SSS/RRRR & SSSSSS/R000 & 0SSSSS/RR00 \\
\hline SSSSSS/RRRR & 0SSSS/RRR00 & 000SS/RRRRR & SSSSS/RR000 & 0SSSS/RRR00 \\
\hline SSSSSS/RRRR & 0SSSSS/RR00 & 000SSS/RRRR & SSSSSS/R000 & 0SSSSS/RR00 \\
\hline SSSSRSSRRR & 0SSSSSS/R00 & 000SSSSS/RR & SSSSSSS000 & 0SSSSSSS00 \\
\hline SSSSSS/RRRR & 0SSSS/RRR00 & 000SSS/RRRR & SSSSSS/R000 & 0SSSSS/RR00 \\
\hline SSSSSSSSS/R & 0SSSSSSS00 & 000SSSSSS/R & SSSSSSS000 & 0SSSSSSS00 \\
\hline SSSSSSSS/RR & 0SSSSSS/R00 & 000SSSSS/RR & SSSSSSS000 & 0SSSSSSS00 \\
\hline SSSSSSSS/RR & 0SSSSSS/R00 & 000SSSSS/RR & SSSSSSS000 & 0SSSSSS/R00 \\
\hline SSSSS/RRRRR & 0SSSS/RRR00 & 000SSS/RRRR & SSSSSS/R000 & 0SSSSSS/R00 \\
\hline
\end{tabular}

Table A6. Choices of subjects (26) in Session $F$ for the Holt and Laury method.

$\begin{array}{lllll}\text { Treatment } 5 & \text { Treatment } 3 & \text { Treatment 2 } & \text { Treatment 1 } & \text { Treatment } 5 \\ \text { SSSRSSR000 } & \text { 00SSRRSRS0 } & \text { 000SSS/RRRR } & \text { SSSSRSRRRS } & \text { SSRSRSR000 } \\ \text { SSS/RRRR000 } & \text { 00S/RRRRRR0 } & \text { 000RRRRRRR } & \text { SSS/RRRRRR } & \text { SSS/RRRR000 } \\ \text { SSSRSRR000 } & \text { 00RRRSRSS0 } & \text { 000SRSRSRS } & \text { RRRSSSSRRR } & \text { SSSS/RRR000 } \\ \text { SSSSS/RR000 } & \text { 00SSS/RRRR0 } & \text { 000SS/RRRRR } & \text { SSSSSRRRRR } & \text { SSSSS/RR000 } \\ \text { SRSSRSS000 } & \text { 00SSSSS/RR0 } & \text { 000SSSS/RRR } & \text { SSSSSSSRRR } & \text { SSSSSSS000 } \\ \text { SSSSS/RR000 } & \text { 00SSSS/RRR0 } & \text { 000SSSS/RRR } & \text { SSSSSSSRRR } & \text { SSSSSSS000 } \\ \text { SSSSSS/R000 } & \text { 00SSSS/RRR0 } & \text { 000SSS/RRRR } & \text { SSSSSSRRRR } & \text { SSSSSS/R000 } \\ \text { SSSSS/RR000 } & \text { 00SSSS/RRR0 } & \text { 000SSS/RRRR } & \text { SSSSSSRRRR } & \text { SSSSSS/R000 } \\ \text { SSSS/RRR000 } & \text { 00SS/RRRRR0 } & \text { 000S/RRRRRR } & \text { SSSSRRRRR } & \text { SSSS/RRR000 } \\ \text { SSSSS/RR000 } & \text { 00SSSS/RRR0 } & \text { 000SSS/RRRR } & \text { SSSSSSRRRR } & \text { SSSSSSR000 } \\ \text { SSSS/RRR000 } & \text { 00SSS/RRRR0 } & \text { 000SS/RRRRR } & \text { SSSSSRRRRR } & \text { SSSSS/RR000 } \\ \text { SSSSSS/R000 } & \text { 00SSSSS/RR0 } & \text { 000SSSSS/RR } & \text { SSSSSSSSRR } & \text { SSSSSSS000 } \\ \text { SSSSS/RR000 } & \text { 00SSSS/RRR0 } & \text { 000S/RRRRRR } & \text { SSSSSRRRRR } & \text { SSSSSS/000 }\end{array}$

Table A7.

Choices of subjects (13) in Session $A i$ (= A with order inverted) for the Holt and Laury method. 


\begin{tabular}{|c|c|c|c|c|}
\hline & & & & \\
\hline & & & & \\
\hline & & & SSSS/RRRR & \\
\hline & & & & \\
\hline & & & & \\
\hline & 00 & & & \\
\hline RRR & 00 & 0 & RR & \\
\hline & & & R & \\
\hline & & & & \\
\hline & & & & \\
\hline RR & 0 & & & \\
\hline $\mathrm{RR}$ & 0 & & & \\
\hline & & & & \\
\hline & & & & 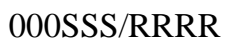 \\
\hline & & & & \\
\hline & P & & & \\
\hline & & & & \\
\hline RR & & & & \\
\hline & & & & \\
\hline & & & & \\
\hline & & 0 & SSI & RSR \\
\hline & & & & RR \\
\hline & & & & \\
\hline & & & & \\
\hline & RR00 & & & \\
\hline RRRR & RR00 & SSSS & SSSS & \\
\hline 00SSS/RRRR & 0SSSSS/RR00 & SSSSSS/R000 & SSSSSS/RRRR & 000SSS/RRR \\
\hline
\end{tabular}

Table A8.

Choices of subjects (26) in Session $B i$ (= B with order inverted) for the Holt and Laury method. 


\begin{tabular}{|c|c|c|c|c|}
\hline & & & & \\
\hline RRR & & RR0 & $\mathrm{R}$ & $\mathrm{S}$ \\
\hline & & & & \\
\hline $\mathrm{RR}$ & & 20 & & RRR \\
\hline & & & & RRR \\
\hline & & & & /RR \\
\hline & & & & RRR \\
\hline & & & & RRRR \\
\hline //RR & & & & S/RR \\
\hline RRR & 0 & R0 & $\mathrm{RR}$ & RRR \\
\hline & & & & RRR \\
\hline & & & & RRR \\
\hline & & & & RR \\
\hline & & & & RRR \\
\hline & & & & RR \\
\hline $\mathrm{R}$ & & & & RR \\
\hline $\mathrm{RR}$ & & & & \\
\hline & & & & \\
\hline & & & & RR \\
\hline RR & sSs & 20 & & RR \\
\hline & & & & RR \\
\hline & & & & RRR \\
\hline $\mathrm{R}$ & 0 & & RR & $\mathrm{RR}$ \\
\hline $\mathrm{R}$ & & & & RRR \\
\hline & & 20 & $\mathrm{RR}$ & S/RR \\
\hline SSS & & & $\mathrm{RR}$ & SSS \\
\hline R/S & & & & RRR \\
\hline & & & & \\
\hline RRR & & & $\mathrm{RR}$ & RRRRR \\
\hline SSSS & 000 & B/RRR0 & $000 \mathrm{~S}$ & SSSSS \\
\hline & & & & SSSS \\
\hline & & & & RRR \\
\hline & & & & SSS \\
\hline SSSSSSS/RR & SSSSS/RR000 & 00SSSSSSS0 & 000SSSSS/RR & SSSSSSSS/RR \\
\hline RRR & SSS/RR000 & SS/RR0 & 000SSSS/RRR & SSSSSSS/RRR \\
\hline SDSS/RKKKK & 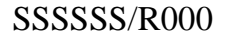 & UUSSSSSRK & UUUSSSTRKI & SSSSSSS/RK1 \\
\hline
\end{tabular}

Table A9.

Choices of subjects (35) in Session $C i$ ( $=C$ with order inverted) for the Holt and Laury method. 


\begin{tabular}{|c|c|c|c|c|}
\hline 3 & ment 5 & Treatment 2 & Treatment 1 & Treatment 3 \\
\hline /RRR0 & S/RRR000 & 000SS/RRRRR & SSSSS/RRRRR & 00SSS/RRRR0 \\
\hline 00SSS/RRRR0 & SSSS/RR000 & RRR & SSSSS/RRRRR & RRR0 \\
\hline DSSSS/RRR0 & SSS/R/S/R000 & $\mathrm{RR}$ & SS/RRRRRRR & 00 SSS/RRRR \\
\hline JSSS/R/SS/R0 & SSSSS/R000 & 000SSSS/RRR & /RRRR & S/RRR0 \\
\hline SSS/RRRR0 & SSSS/RR000 & 000SS/RRRRR & SS/RRRRRR & SS/RRRRRC \\
\hline 0SS/R/S/RRR0 & SSS/R/S/R000 & 000SSS/RRRR & SSSSSS/RRRR & RRR \\
\hline 00SSSS/RRR0 & SSSSS/RR000 & 000SSS/RRRR & SSSSSS/RRRR & 00SS \\
\hline S/RRR0 & SSSSS/R000 & $\mathrm{RR}$ & SSSSSS/RRR & 00SSSS/RRR \\
\hline ORRRRRRR0 & SSSSSS000 & 00SS/RRRRR & SSSS/RRRRRR & 00SS/RRRRR0 \\
\hline RR0 & 00 & 000SSS/RRRR & $R R$ & RRO \\
\hline R0 & 000 & 000SSSS/RRR & SSSSSSS/RRR & 00SSSSS/RR0 \\
\hline 0SSSS/RRR0 & SSSS/RR000 & RRR & RRRR & 00SS \\
\hline RR0 & 00 & RR & RRR & 00SS \\
\hline OSSSS/RRR0 & SSSSS/R000 & 000Ss & SSSSSS/RRRR & 00SSSS/RRR0 \\
\hline 00SSSS/RRR0 & SSSSS/R000 & 000SSS/RRRR & SSSSSSS/RRR & 00SSSSS/RR0 \\
\hline RRR0 & 000 & 000S/RI & $\mathrm{RR}$ & RRR0 \\
\hline 00SSSS/RRR0 & SSSSS/RR000 & 000SSS/RRRR & SSS & S/RRR0 \\
\hline 00SSS/RRRR0 & SSS/R/S/RR000 & 000S/ & SSSS/RRRRRR & 00SS/RRRRR \\
\hline 00SSSSS/RR0 & SSSSSS000 & 000Ss & SSSSSSS/RRR & 00SSSSS/RR \\
\hline RR0 & R000 & 000SSSS/RRR & /RRRR & 00SSSSS/RR0 \\
\hline /RRR0 & SSSSS/R000 & 000SSSS/RRR & SSSSSS/RRRR & 00SSSSS/RR0 \\
\hline 00SSSSS/RR0 & R/SSS/RR/S000 & $000 \mathrm{SS} / \mathrm{R} / \mathrm{S} / \mathrm{RR} / \mathrm{S}$ & SSSSSS/RRRR & 00SSSSS/RR0 \\
\hline 00SSSSS/RR0 & SS/RRRR000 & 000S/RRRRRR & SSSS/RRRRRR & 00SS/RRRRR \\
\hline 00SSSS/RRR0 & SSSSS/RR000 & 000SSSS/RRR & SSSSSS/RRRR & 00SS/RRRRR \\
\hline
\end{tabular}

Table A10.

Choices of subjects (24) in Session $\mathrm{Di}$ (= D with order inverted) for the Holt and Laury method. 


Treatment 4
0SSSSS/RR00
0SSSS/RRR00
0SSSSSSS00
0SSSSS/RR00
0SSSS/RRR00
0SSSS/RRR00
0SSS/RRRR00
0SS/RR/SS/R00
0SSSSSS/R00
0SSSSS/RR00
0SSSS/RRR00
0SSSS/RRR00
0SSSS/RRR00
0SSSS/RRR00
0SSSS/RRR00
0SSSSS/RR00
0SSSSS/RR00
0SSSSSS/R00
0SSS/RRRR00
0SSSS/RRR00
0RRRRRRR00
0SSSSS/RR00
0SSSS/RRR00
0S/R/A/RRR/A00
0SSSSS/RR00
0SSSSSSS00
0SSSSS/RR00
0SSSSS/RR00

Treatment 1
SSSSSS/RRRR
SSSSSSSS/RR
SSSSSSSS/RR
SSSSSS/RRRR
SSSSS/RRRRR
SSSSSS/RRRR
SSSSS/RRRRR
R/S/R/SSS/R/S/RR
SSSSSS/RRRR
SSSSSS/RRRR
SSSSS/RRRRR
SSSSS/RRRRR
SSSSSS/RRRR
SSSSSS/RRRR
SSSSSS/RRRR
SSSSSSS/RRR
SSSSSSS/RRR
SSSSSSS/RRR
SSSSSS/RRRR
SSSSS/RRRRR
RRRRRRRRRR
SSSSS/RRRRR
SSSSS/RRRRR
SSSS/R/S/RR/S/R
SSSSSS/RRRR
SSSSSS/RRRR
SSSSSS/RRRR
SSSSSS/RRRR

$\begin{array}{ll}\text { Treatment } 2 & \text { Treatment } 3 \\ \text { 000SSSS/RRR } & \text { 00SSSSS/RR0 } \\ \text { 000SSSSS/RR } & \text { 00SSSSSS/R0 } \\ \text { 000SSSSS/RR } & \text { 00SSSSSS/R0 } \\ \text { 000SSSS/RRR } & \text { 00SSSSS/RR0 } \\ \text { 000SSS/RRRR } & \text { 00SSSS/RRR0 } \\ \text { 000SSSS/RRR } & \text { 00SSSSS/RR0 } \\ \text { 000S/RRRRRR } & \text { 00SSS/RRRR0 } \\ \text { 000R/SS/R/SS/R } & \text { 00S/R/S/R/S/RR0 } \\ \text { 000SSSS/RRR } & \text { 00SSSSS/RR0 } \\ \text { 000SSS/RRRR } & \text { 00SSSS/RRR0 } \\ \text { 000SS/RRRRR } & \text { 00SSS/RRRR0 } \\ \text { 000SSS/RRRR } & \text { 00SS/RRRRR0 } \\ \text { 000SSS/RRRR } & \text { 00SSSS/RRR0 } \\ \text { 000SSS/RRRR } & \text { 00SSSS/RRR0 } \\ \text { 000SSS/RRRR } & \text { 00SSSS/RRR0 } \\ \text { 000SSSS/RRR } & \text { 00SSSSS/RR0 } \\ \text { 000SSSS/RRR } & \text { 00SSSSS/RR0 } \\ \text { 000SSSS/RRR } & \text { 00SSSSS/RR0 } \\ \text { 000SSS/RRRR } & \text { 00SSS/RRRR0 } \\ \text { 000RRRRRRR } & \text { 00SSS/RRRR0 } \\ \text { 000RRRRRRR } & \text { 00RRRRRRR0 } \\ \text { 000SS/RRRRR } & \text { 00SSS/RRRR0 } \\ \text { 000SSS/RRRR } & \text { 00SS/RRRRR0 } \\ \text { 000SSS/R/S/RR } & \text { 00SS/RRR/SS0 } \\ \text { 000R/SS/RRRR } & \text { 00SS/RRRRR0 } \\ \text { 000SSSS/RRR } & \text { 00SSSSS/RR0 } \\ \text { 000SSSS/RRR } & \text { 00SSSSS/RR0 } \\ \text { 000SSS/RRRR } & \text { 00SSSS/RRR0 }\end{array}$

Treatment 4
0SSSSSS/R00
0SSSSSS00
0SSSSSS/R00
0SSSSS/RR00
0SSSSS/RR00
0SSSSSS/R00
0SSSS/RRR00
0S/R/S/R/S/R/S00
0SSSSSS/R00
0SSSSS/RR00
0SSSS/RRR00
0SSSS/RRR00
0SSSSS/RR00
0SSSSS/RR00
0SSSSS/RR00
0SSSSSS/R00
0SSSSS/RR00
0SSSSSS/R00
0SSSS/RRR00
0SSS/RRRR00
0RRRRRRR00
0SSSS/RRR00
0SSS/RRRR00
0SS/RR/S/RR00
0SSSS/RRR00
0SSSSSS/R00
0SSSSSS/R00
0SSSSS/RR00

Table A11.

Choices of subjects (28) in Session Ei (= E with order inverted) for the Holt and Laury method.

$\begin{array}{lllll}\text { Treatment 1 } & \text { Treatment } 4 & \text { Treatment 2 } & \text { Treatment 5 } & \text { Treatment } 4 \\ \text { SSSSSS/RRRR } & \text { 0SSSSS/RR00 } & \text { 000SSSS/RRR } & \text { SSSSSSS000 } & \text { 0SSSSSS/R00 } \\ \text { SSSSS/RRRRR } & \text { 0SSS/RRRR00 } & \text { 000SSS/RRRR } & \text { SSSSS/RR000 } & \text { 0SSSSS/RR00 } \\ \text { SSSSS/RRRRR } & \text { 0SSSS/RRR00 } & \text { 000SSS/RRRR } & \text { SSSS/RRR000 } & \text { 0SSSS/RRR00 } \\ \text { SSSSSS/RRRR } & \text { 0SSSSS/RR00 } & \text { 000SSS/RRRR } & \text { SSSSSS/R000 } & \text { 0SSSSS/RR00 } \\ \text { SSSSSS/RRRR } & \text { OSSSS/RRR00 } & \text { 000SS/RRRRR } & \text { SSSSS/RR000 } & \text { 0SSSS/RRR000 } \\ \text { SSSS/RRRRRR } & \text { 0SSS/RRRR00 } & \text { 000SS/RRRRR } & \text { SSSSSS/R000 } & \text { 0SSSS/RRR00 } \\ \text { SSSSSSS/RRR } & \text { 0SSSSSS/R00 } & \text { 000SSSS/RRR } & \text { SSSSSSS000 } & \text { 0SSSSSS/R00 } \\ \text { SSSSSSS/RRR } & \text { 0SSSSSS/R00 } & \text { 000SSSS/RRR } & \text { SSSSSSS000 } & \text { 0SSSSSS/R00 } \\ \text { SSSSSS/RRRR } & \text { 0SSSS/RRR00 } & \text { 000SS/RRRRR } & \text { SSSSS/RR000 } & \text { 0SSSS/RRR00 } \\ \text { SSSSSSSS/RR } & \text { 0SSSSS/RR00 } & \text { 000SSSS/RRR } & \text { SSSSSS/R000 } & \text { 0SSSSS/RR00 } \\ \text { SSSSSS/RRRR } & \text { 0SSSS/RRR00 } & \text { 000SSS/RRRR } & \text { SSSS/RRR000 } & \text { 0SSSS/RRR00 } \\ \text { SSSS/RRRRRR } & \text { 0SSS/RRRR00 } & \text { 000SSS/RRRR } & \text { SSSSS/RR000 } & \text { 0SSSSS/RR00 } \\ \text { SSSSSS/RRRR } & \text { 0SS/RR/SSS00 } & \text { 000SS/RRRRR } & \text { SSSSSSS000 } & \text { 0SSSSS/RR00 }\end{array}$

Table A12.

Choices of subjects (13) in Session Fi (=F with order inverted) for the Holt and Laury method. 


$\begin{array}{lllll}\text { Treatment } 1 & \text { Treatment } 5 & \text { Treatment } 3 & \text { Treatment } 4 & \text { Treatment } 1 \\ \text { RR/S/RR/SSSS } & \text { RRRR/SSS000 } & \text { 00RRR/SSSS0 } & \text { 0RR/SSSSS00 } & \text { RRR/SSSSSSS } \\ \text { RRRR/SSSSSS } & \text { RRRR/SSS000 } & \text { 00RR/SSSSS0 } & \text { 0RRR/SSSS00 } & \text { RRRR/SSSSSS } \\ \text { RRR/SSSSSSS } & \text { RRR/SSSS000 } & \text { 00R/SSSSSS0 } & \text { 0RR/SSSSS00 } & \text { RRR/SSSSSSS } \\ \text { RRR/SSSSSSS } & \text { RRR/SSSS000 } & \text { 00RR/SSSSS0 } & \text { 0RR/SSSSS00 } & \text { RRR/SSSSSSS } \\ \text { S/RRRR/SSSSS } & \text { RRRRR/SS000 } & \text { 00RRR/SSSS0 } & \text { 0RRRR/SSS00 } & \text { RRRRR/SSSSS } \\ \text { RRR/SSSSSSS } & \text { RRR/SSSS000 } & \text { 00RR/SSSSS0 } & \text { 0RR/SSSSS00 } & \text { RRRR/SSSSSS } \\ \text { RRRR/SSSSSS } & \text { RRRR/SSS000 } & \text { 00RR/SSSSS0 } & \text { 0RRR/SSSS00 } & \text { RRRR/SSSSSS } \\ \text { RRRRR/SSSSS } & \text { RRRRR/SS000 } & \text { 00RR/SSSSS0 } & \text { 0RRRR/SSS00 } & \text { RRRRR/SSSSS } \\ \text { RR/SSSSSSSS } & \text { RR/SSSSS000 } & \text { 00SSSSSSS0 } & \text { 0R/SSSSSS00 } & \text { RR/SSSSSSSS } \\ \text { RRRR/SSSSSS } & \text { RRRR/SSS000 } & \text { 00RR/SSSSS0 } & \text { 0RRR/SSSS00 } & \text { RRRR/SSSSSS } \\ \text { RRRR/SSSSSS } & \text { RRRR/SSS000 } & \text { 00RR/SSSSS0 } & \text { 0RRR/SSSS00 } & \text { RRRR/SSSSSS } \\ \text { RRRR/SSSSSS } & \text { RRRR/SSS000 } & \text { 00RR/SSSSS0 } & \text { 0RRR/SSSS00 } & \text { RRRR/SSSSSS } \\ \text { RRRR/SSSSSS } & \text { RRRR/SSS000 } & \text { 00RR/SSSSS0 } & \text { 0RRR/SSSS00 } & \text { RRRR/SSSSSS } \\ \text { RRRRR/SSSSS } & \text { RRRR/SSS000 } & \text { 00RRR/SSSS0 } & \text { 0RRRR/SSS00 } & \text { RRRRR/SSSSS } \\ \text { RRRR/SSSSSS } & \text { RRRR/SSS000 } & \text { 00RR/SSSSS0 } & \text { 0RRR/SSSS00 } & \text { RRRR/SSSSSS } \\ \text { RRRRR/SSSSS } & \text { RRRRR/SS000 } & \text { 00RRR/SSSS0 } & \text { 0RRRR/SSS00 } & \text { RRRRR/SSSSS } \\ \text { RRRRRR/SSSS } & \text { RRRRRR/S000 } & \text { 00RRRRR/SS0 } & \text { 0RRRRRR/S00 } & \text { RRRRRR/SSSS } \\ \text { RRR/SSSSSSS } & \text { RRR/SSSS000 } & \text { 00RR/SSSSS0 } & \text { 0RR/SSSSS00 } & \text { RRR/SSSSSSS } \\ \text { RRR/SSSSSSS } & \text { RRRR/SSS000 } & \text { 00RR/SSSSS0 } & \text { 0RR/SSSSS00 } & \text { RRR/SSSSSSS } \\ \text { RRRRR/SSSSS } & \text { RRRRR/SS000 } & \text { 00RRR/SSSS0 } & \text { 0RRRR/SSS00 } & \text { RRRRR/SSSSS } \\ \text { RRRR/SSSSSS } & \text { RRRR/SSS000 } & \text { 00RRR/SSSS0 } & \text { 0RRRR/SSS00 } & \text { RRRRR/SSSSS }\end{array}$

Table A13.

Choices of subjects (21) in Session A for the Abdellaoui et al. method. 


\begin{tabular}{|c|c|c|c|c|}
\hline & & & & \\
\hline 5 & S & & 0 & \\
\hline & & & 00 & \\
\hline & & & & \\
\hline & & & & \\
\hline & & & & \\
\hline & & & & \\
\hline & & & & \\
\hline$S$ & & & 00 & \\
\hline & & & & \\
\hline & & & & \\
\hline & & & & \\
\hline & & & & \\
\hline & & & & \\
\hline SS & SS & & 00 & SSS \\
\hline SSS & RRR & 500 & 00 & SSSS \\
\hline SS & & & & \\
\hline & & & & \\
\hline CS & SS & ORI & & 0001 \\
\hline 000SS/F & SSSSS/ & ORF & 00 & 000 \\
\hline SSS & SS & $0 \mathrm{R}$ & 00 & 000S \\
\hline 00SSSSSSS & RR/SSSSSSSS & 0R/SSSSSS00 & RR/SSSSS000 & 000SSSSSSS \\
\hline
\end{tabular}

Table A14. Choices of subjects (21) in Session B for the Abdellaoui et al. method. 


\begin{tabular}{|c|c|c|c|c|}
\hline & & & & \\
\hline 0 & $\mathrm{~s}$ & 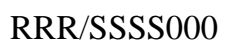 & S & SSS0 \\
\hline 0 & 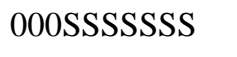 & & SS & 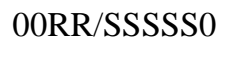 \\
\hline & 000 & & SSS & S0 \\
\hline SS0 & $000 s$ & SS000 & SSSS & SSO \\
\hline 50 & 000 & & $S$ & 0 \\
\hline & & & & \\
\hline & & & & \\
\hline R & 0001 & 00 & SS & $00 \mathrm{R}$ \\
\hline So & $000 \mathrm{~s}$ & 00 & $\mathrm{RR} /$ & 00Ss \\
\hline SO & 000 & 00 & SS & SSO \\
\hline 30 & $000 \mathrm{~F}$ & 0 & SS & SO \\
\hline SO & 0001 & & & \\
\hline n & & & & \\
\hline 30 & 000F & 0 & SS & 00R \\
\hline ORR & 000Ss & 00 & $\mathrm{RR}$ & 00R \\
\hline 0 & $000 \mathrm{~F}$ & 0 & SS & SS0 \\
\hline OR & 000R & & & \\
\hline $00 \mathrm{R} /$ & $000 \mathrm{R} /$ & S000 & SS & 00RF \\
\hline ORRR & 000R/SSSSSS & RRR & RRR & 00RR \\
\hline ORR/SSSSS0 & 000R/SSSSSS & RRRR/SSS000 & RRRR/SSSSSS & 00RR/SSSSS0 \\
\hline
\end{tabular}

Table A15. Choices of subjects (20) in Session $C$ for the Abdellaoui et al. method. 


\begin{tabular}{|c|c|c|c|c|}
\hline & & & & \\
\hline & & & & \\
\hline & & & & \\
\hline & & & & \\
\hline & 50 & & & \\
\hline 00 & $0 \mathrm{RI}$ & SS & $S$ & \\
\hline & & & & \\
\hline & & & & \\
\hline 0 & 00RF & & & \\
\hline 00 & 0 & & $S$ & \\
\hline 00 & 0 & & $S$ & \\
\hline & SO & SS & 000 & 500 \\
\hline 0 & 0 & & & \\
\hline & & & & \\
\hline & & & & \\
\hline & 0 & & 000 & 00 \\
\hline $\mathrm{RR}$ & $00 \mathrm{R} /$ & & 00 & 500 \\
\hline & 0 & & SS & 00 \\
\hline & 00RF & & 000R & \\
\hline 00 & 00RP & & & \\
\hline ORR & 00RR/SSSS & SSS & 000R/SSSSSS & \\
\hline $\mathrm{P} / \mathrm{C}$ & DR/SSSSSS0 & RRR/SSSSSSS & 000SSSSSSS & 0RR/SSS \\
\hline
\end{tabular}

Table A16. Choices of subjects (21) in Session $D$ for the Abdellaoui et al. method. 


\begin{tabular}{|c|c|c|c|c|}
\hline nt 5 & Tre & nt 2 & Treatment 3 & t 5 \\
\hline 0 & 0 & 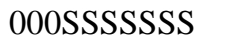 & 0 & 0 \\
\hline SSS000 & 0RRR/SSSS00 & 00R/SSSSSS & RR/SSSSS0 & RRRR/SSS000 \\
\hline $\mathrm{RRR} / \mathrm{S} / \mathrm{R} / \mathrm{SOOC}$ & 0R/S/R/SSS/R00 & 000SS/R/S/R/SS & 00S/RRR/SS/R0 & $\mathrm{RR} / \mathrm{SS} / \mathrm{R} / \mathrm{S} / \mathrm{R} / 000$ \\
\hline RRRRR/SS000 & 0RRRR/SSS00 & 000RR/SSSSS & 00RRR/SSSS0 & RRRRR/SS000 \\
\hline RRR/SSSS000 & 0RRR/SSSS00 & $000 \mathrm{R} / \mathrm{s}$ & 00RI & RRR \\
\hline 000 & OR/SS & $000 \mathrm{~S}$ & 0 & $\mathrm{RR} / \mathrm{S}$ \\
\hline 00 & ORR & & & 000 \\
\hline RRF & ORR & $S$ & SO & 000 \\
\hline RRRR/SSS000 & 0RRRRR/SS00 & 000RRR/SSSS & 00RRRR/SSS0 & RRRRR/SS000 \\
\hline RRR/SS/RR000 & 0RRR/SSSS00 & 000RRR/SSSS & 00RR/SSSSS0 & RRRRR/SS000 \\
\hline RRR/SSSS000 & 0RRRR/SSS00 & 000R/SSSSSS & 00RR/SSSSS0 & RRRRR/SS000 \\
\hline $\mathrm{RR} / \mathrm{S}$ & ORR/ & SS & SSO & RRR/SSSS000 \\
\hline $\mathrm{RRR} / \mathrm{S}$ & 0RR/S & $000 \mathrm{~S}$ & $00 \mathrm{R}$ & RRF \\
\hline $\mathrm{RR} / \mathrm{SS}$ & ORR/SS & $000 \mathrm{~S}$ & $00 \mathrm{R} / 4$ & $\mathrm{RRR} / \mathrm{s}$ \\
\hline RRRRR/SS000 & 0RRRR/SSS00 & 000RR/SSSSS & 00RRR/SSSS0 & RRRRR/SS000 \\
\hline RRRR/SSS000 & 0RRR/SSSS00 & 000R/SSSSSS & 00RR/SSSSS0 & RRRR/SSS000 \\
\hline RRR/SSSS000 & 0RR/SSSSS00 & 000R/SSSSSS & 00R/SSSSSS0 & RRR/SSSS000 \\
\hline RRRRR/SS000 & 0RRRR/SSS00 & 000RR/SSSSS & 00RRR/SSSS0 & RRRRR/SS000 \\
\hline RRRRR/SS000 & 0RRRR/SSS00 & 000RR/SSSSS & 00RRR/SSSS0 & RRRRR/SS000 \\
\hline RRRR/SSS000 & 0RRR/SSSS00 & 000R/SSSSSS & 00RR/SSSSS0 & RRRR/SSS000 \\
\hline RRRR/SSS000 & 0RRR/SSSS00 & 000R/SSSSSS & 00RRR/SSSS0 & RRRR/SSS000 \\
\hline
\end{tabular}

Table A17. Choices of subjects (21) in Session $E$ for the Abdellaoui et al. method. 


\section{Appendix B}

Let the decision maker face the complete list of ten pairs in the HL method. Disregarding compound choices that switch back and forth, and are therefore first-order stochastically dominated, there are eleven possible compound choices, listed in the first column of Table B1, with their expected values listed in the last column. Assuming that one of the ten lottery pairs is randomly picked, the cumulative distribution functions (CDF's) corresponding to each of the compound choices are given by columns 2 to 5 of Table B1. ${ }^{12}$

\begin{tabular}{|l|l|l|l|l|l|}
\hline $\begin{array}{l}\text { Compound } \\
\text { Choice }\end{array}$ & $€ \mathbf{0 . 3 0}$ & $€ 4.80$ & $€ 6.00$ & $€ \mathbf{1 1 . 5 5}$ & $\begin{array}{l}\text { Expected } \\
\text { Money Value } \\
(€)\end{array}$ \\
\hline SSSSSSSSSS & 0 & 0.45 & 1.00 & 1.00 & 5.46 \\
\hline SSSSSSSSS/R & 0 & 0.45 & 0.90 & 1.00 & 6.01 \\
\hline SSSSSSSS/RR & 0.01 & 0.45 & 0.81 & 1.00 & 6.47 \\
\hline SSSSSSS/RRR & 0.03 & 0.45 & 0.73 & 1.00 & 6.82 \\
\hline SSSSSS/RRRR & 0.06 & 0.45 & 0.66 & 1.00 & 7.08 \\
\hline SSSSS/RRRRR & 0.10 & 0.45 & 0.60 & 1.00 & 7.23 \\
\hline SSSS/RRRRRR & 0.15 & 0.45 & 0.55 & 1.00 & 7.28 \\
\hline SSS/RRRRRRR & 0.21 & 0.45 & 0.51 & 1.00 & 7.23 \\
\hline SS/RRRRRRRR & 0.28 & 0.45 & 0.48 & 1.00 & 7.09 \\
\hline S/RRRRRRRRR & 0.36 & 0.45 & 0.46 & 1.00 & 6.84 \\
\hline RRRRRRRRRR & 0.45 & 0.45 & 0.45 & 1.00 & 6.49 \\
\hline
\end{tabular}

Table B1. CDF's for the eleven choices of the full ten-pair list in HL.

On the other hand, if the last three pairs are deleted, eight compound choices are available, as follows. (Recall that zeroes in the compound choice column mark the deletion of pairs.)

\footnotetext{
${ }^{12}$ We adopt the consequentialist axiom, see Mas-Colell et al. (1995).
} 


\begin{tabular}{|l|l|l|l|l|l|}
\hline $\begin{array}{l}\text { Compound } \\
\text { Choice }\end{array}$ & $\boldsymbol{€ 0 . 3 0}$ & $\mathbf{€ 4 . 8 0}$ & $\boldsymbol{€ 6 . 0 0}$ & $\mathbf{€ 1 1 . 5 5}$ & $\begin{array}{l}\text { Expected } \\
\text { Money Value } \\
(€)\end{array}$ \\
\hline SSSSSSS/000 & 0.00 & 0.60 & 1.00 & 1.00 & 5.28 \\
\hline SSSSSS/R/000 & 0.04 & 0.60 & 0.90 & 1.00 & 5.64 \\
\hline SSSSS/RR/000 & 0.10 & 0.60 & 0.81 & 1.00 & 5.86 \\
\hline SSSS/RRR/000 & 0.17 & 0.60 & 0.74 & 1.00 & 5.94 \\
\hline SSS/RRRR/000 & 0.26 & 0.60 & 0.69 & 1.00 & 5.87 \\
\hline SS/RRRRR/000 & 0.36 & 0.60 & 0.64 & 1.00 & 5.66 \\
\hline S/RRRRRR/000 & 0.47 & 0.60 & 0.61 & 1.00 & 5.30 \\
\hline RRRRRRR/000 & 0.60 & 0.60 & 0.60 & 1.00 & 4.80 \\
\hline
\end{tabular}

Table B2. CDF's for the eight choices of the seven-pair list after the deletion of the last three pairs in HL.

It can be easily checked that each of the eleven compound choices in the ten-pair list (Table B1) first-order stochastically dominates (FOSD) one, two, three, four, five or six of the compound choice in the seven pair (eight choices) list of Table B2, as follows.

SSSSSSSSSS FOSD SSSSSSS/000;

SSSSSSSSS/R FOSD SSSSSS/R/000 and SSSSSSS/000;

SSSSSSS/RR FOSD SSSSS/RR/000 and SSSSSS/R/000;

SSSSSSS/RRR FOSD SSSS/RRR/000, SSSSS/RR/000 and SSSSSS/R/000;

SSSSSS/RRRR FOSD SSS/RRRR/000, SSSS/RRR/000 and SSSSS/RR/000;

SSSSS/RRRRR FOSD RRRRRRR/000, S/RRRRRR/000, SS/RRRRR/000, SSS/RRRR/000, $S S S S / R R R / 000$ and $S S S S S / R R / 000$;

SSSS/RRRRRR FOSD RRRRRRR/000, S/RRRRRR/000, SS/RRRRR/000, SSS/RRRR/000 and $S S S S / R R R / 000$;

SSS/RRRRRRR FOSD RRRRRRR/000, S/RRRRRR/000, SS/RRRRR/000 and SSS/RRRR/000; $S S / R R R R R R R R$ FOSD RRRRRRR/000, S/RRRRRR/000 and SS/RRRRR/000; $S / R R R R R R R R R$ FOSD RRRRRRR/000, S/RRRRRR/000 and SS/RRRRR/000; RRRRRRRRRR FOSD RRRRRRR/000 and S/RRRRRR/000. 
The corresponding CDF's and expected money values for the full ten-pair list of the ADH method are as follows

\begin{tabular}{|c|c|c|c|c|c|c|c|c|c|c|c|}
\hline $\begin{array}{l}\text { Compound } \\
\text { Choice }\end{array}$ & $€ 5$ & $€ 6.10$ & $€ 7.20$ & $€ 8.30$ & $€ 9.45$ & $€ 10.55$ & $€ 11.70$ & $€ 12.80$ & $€ 13.90$ & $€ 15$ & $\begin{array}{l}\text { Expected } \\
\text { Money } \\
\text { Value } \\
(€)\end{array}$ \\
\hline RRRRRRRRRR & 0.50 & 0.50 & 0.50 & 0.50 & 0.50 & 0.50 & 0.50 & 0.50 & 0.50 & 1.00 & 10 \\
\hline RRRRRRRRR/S & 0.45 & 0.45 & 0.45 & 0.45 & 0.45 & 0.45 & 0.45 & 0.45 & 0.45 & 1.00 & 10.50 \\
\hline RRRRRRRR/SS & 0.40 & 0.40 & 0.40 & 0.40 & 0.40 & 0.40 & 0.40 & 0.40 & 0.50 & 1.00 & 10.89 \\
\hline$R R R R R$ & 0.35 & 0.35 & 35 & 0.35 & 0.35 & .35 & 130 & .45 & 0.55 & 1.00 & 11.17 \\
\hline RRRRRR/SSSS & 0.30 & 0.30 & 0.30 & 0.30 & 0.30 & 0.30 & 0.40 & 0.50 & 0.60 & 1.00 & 11.34 \\
\hline RRRRR/SSSSS & 0.25 & 0.25 & 0.25 & 0.25 & 0.25 & 0.35 & 0.45 & 0.55 & 0.65 & 1.001 .0 & 11.40 \\
\hline$R R R R / S S S S S S$ & 0.20 & 0.20 & 0.20 & 0.20 & 0.30 & 0.40 & 0.50 & 0.60 & 0.70 & 1.00 & 11.34 \\
\hline$R R R / S S S S S S S$ & 0.15 & 0.15 & 0.15 & 0.25 & 0.35 & 0.45 & 0.55 & 0.65 & 0.75 & 1.00 & 11.17 \\
\hline RR/SSSSSSSS & 0.10 & 0.10 & 0.20 & 0.30 & 0.40 & 0.50 & 0.60 & 0.70 & 0.80 & 1.00 & 11.89 \\
\hline R/SSSSSSSSS & 0.05 & 0.15 & 0.25 & 0.35 & 0.45 & 0.55 & 0.65 & 0.75 & 0.85 & 1.00 & 10.50 \\
\hline SSSSSSSSSS & 0.10 & 0.20 & 0.30 & $\theta .4 \theta$ & 0.50 & $\theta .60$ & $\theta .70$ & $\theta .80$ & $\theta .90$ & 1.00 & 10 \\
\hline
\end{tabular}

Table B3. CDF's for the compound choices of the full ten-pair list in the ADH method.

Note that, in Table B3, the compound choice RRRRRRRRRR is first-order stochastically dominated (by RRRRRRRRR/S) and so is SSSSSSSSSS (by R/SSSSSSSSS and by RR/SSSSSSSS); accordingly, they are disregarded.

Again, if the last three pairs are deleted, eight compound choices are available, as follows. 


\begin{tabular}{|c|c|c|c|c|c|c|c|c|c|c|c|}
\hline $\begin{array}{l}\text { Compound } \\
\text { Choice }\end{array}$ & $€ 5$ & $€ 6.10$ & $€ 7.20$ & $€ 8.30$ & $€ 9.45$ & $€ 10.55$ & $€ 11.70$ & $€ 12.80$ & $€ 13.90$ & $€ 15$ & \begin{tabular}{|l|} 
Expected \\
Money \\
Value $(€)$
\end{tabular} \\
\hline RRRRRRR/000 & 0.50 & 0.50 & 0.50 & 0.50 & 0.50 & 0.50 & 0.50 & 0.50 & 0.50 & 1.00 & 10 \\
\hline RRRRRR/S/000 & 0.43 & 0.43 & 0.43 & 0.43 & 0.43 & 0.43 & 0.57 & 0.57 & 0.57 & 1.00 & 10.24 \\
\hline$R R R F$ & 0.36 & 0.36 & 0.36 & 0.36 & 36 & .5 & 64 & 0.64 & 0.64 & \begin{tabular}{|l|}
1.00 \\
\end{tabular} & 10.32 \\
\hline$R R R R / S S S / 000$ & 0.29 & 0.29 & 0.29 & 0.29 & 0.43 & 0.57 & 0.71 & 0.71 & 0.71 & 1.00 & 10.24 \\
\hline RRR/SSSS/000 & 0.21 & 0.21 & 0.21 & 0.36 & 0.50 & 0.64 & 0.79 & 0.79 & 0.79 & 1.00 & 10 \\
\hline$R R / S S S S S / 000$ & 0.14 & 0.14 & 0.29 & 0.43 & 0.57 & 0.71 & 0.86 & 0.86 & 0.86 & 1.00 & 9.60 \\
\hline$R / S S S S S S / 000$ & 0.07 & 0.21 & 0.36 & 0.50 & 0.64 & 0.79 & 0.93 & 0.93 & 0.93 & \begin{tabular}{|l|}
1.00 \\
\end{tabular} & 9.04 \\
\hline SSSS & .14 & 0.29 & 0.43 & 0.57 & 0.71 & 0.86 & 1.00 & 1.00 & 1.00 & 1.00 & 8.33 \\
\hline
\end{tabular}

Table B4. CDF's for the compound choices of the seven-pair list after the deletion of the last three pairs in the ADH method.

In Table B4, the compound choice SSSSSSS/000 is first-order stochastically dominated (by $R / S S S S S S / 000$ and by $R R / S S S S S / 000$ ), and is disregarded.

Now each of the nine compound choices in Table B3 FOSD one or two of the seven compound choices in Table B2, as follows.

$R R R R R R R R R / S$ FOSD RRRRRRR/000;

$R R R R R R R R / S S$ FOSD RRRRRR/S/000 and $R R R R R R R / 000$;

$R R R R R R R / S S S$ FOSD RRRRR/SS/000 and RRRRRR/S/000;

RRRRRR/SSSS FOSD RRRRR/SS/000;

$R R R R R /$ SSSSS FOSD RRRR/SSS/000;

$R R R R / S S S S S S$ FOSD RRR/SSSS/000 and RRRR/SSS/000;

$R R R / S S S S S S S$ FOSD RRR/SSSS/000;

$R R / S S S S S S S S$ FOSD RR/SSSSS/000;

$R / S S S S S S S S S$ FOSD R/SSSSSS/000. 


\section{REFERENCES}

Abdellaoui, Mohammed, Ahmed Driouchi and Olivier L’Haridon (2011). "Risk Aversion Elicitation: Reconciling Tractability and Bias Minimization.” Theory and Decision 71: 63-80.

Amaldoss, Wilfred, James R. Bettman and John W. Payne (2008). "Biased but Efficient: An Investigation of Coordination Facilitated by Asymmetric Dominance.” Marketing Science 27: 903-921.

Andersen, Steffen, Glenn W. Harrison, Morten Igel Lau and E. Elisabet Rutström (2006). “Elicitation Using Multiple Price List Formats.” Experimental Economics 9(4): 383-405.

Bateman, Ian, Brett Day, Graham Loomes and Robert Sugden (2007). "Can Ranking Techniques Elicit Robust Values?” Journal of Risk and Uncertainty 34: 49-66.

Beauchamp, Jonathan P., Daniel J. Benjamin, Christopher F. Chabris and David I. Laibson (2012). “How Malleable Are Risk Preferences and Loss Aversion?” WP.

Bosch-Domènech, Antoni, and Joaquim Silvestre (1999). "Does Risk Aversion or Attraction Depend on Income? An Experiment.” Economics Letters 65: 265-273.

Bosch-Domènech, Antoni, and Joaquim Silvestre (2006a). "Do the Wealthy Risk More Money? An Experimental Comparison.” In Christian Schultz and Karl Vind, eds., Institutions, Equilibria and Efficiency: Essays in Honor of Birgit Grodal: 95-106, Berlin: Springer-Verlag.

Bosch-Domènech, Antoni, and Joaquim Silvestre (2006b). "Reflections on Gains and Losses: A 2x2x7 Experiment.” Journal of Risk and Uncertainty 33(3): 217-235.

Bosch-Domènech, Antoni, and Joaquim Silvestre (2006c). "The Gain-Loss Asymmetry and Single-Self Preferences.” In Shigeo Kusuoka and Akira Yamazaki, eds., Advances in Mathematical Economics, Berlin: Springer Verlag.

Bosch-Domènech, Antoni, and Joaquim Silvestre (2010). “Averting Risk in the Face of

Large Losses: Bernoulli vs. Tversky and Kahneman.” Economics Letters 107(2): 180-182.

Bruhin, Adrian, Helga Fehr-Duda and Thomas Epper (2010). "Risk and Rationality:

Uncovering Heterogeneity in Probability Distortion.” Econometrica 78-4, 1375-1412.

Dave, Chetan, Catherine C. Eckel, Cathleen A. Johnson and Christian Rojas (2010).

“Eliciting Risk Preferences: When Is Simple Better?” Journal of Risk and Uncertainty 41: 219243.

Drichoutis, Andreas, and Jayson Lusk (2012). "Risk Preference Elicitation without the Confounding Effect of Probability Weighting.” MPRA Paper No. 37776.

Farquhar, Peter H. (1984). "Utility Assessment Methods.” Management Science 30-11, 1283-1300.

Fehr-Duda, Helga, Adrian Bruhin and Thomas Epper (2010). "Rationality on the Rise:

Why Relative Risk Aversion Increases with Stake Size.” Journal of Risk and Uncertainty 40, 147180.

Fehr-Duda, Helga and Thomas Epper (2012). "Probability and Risk: Foundations and Economic Implications of Probability-Dependent Risk Preferences.” Annual Review of Economics 4, 19.1-19.27.

Harrison, Glenn W., Eric Johnson, Melayne M. McInnes and E. Elisabet Rutström (2005).

“Risk Aversion and Incentive Effects: Comment.” American Economic Review 95(3): 897-901.

Hershey, John C., Howard C. Kunreuther and Paul J. H. Schoemaker (1982). "Sources of

Bias in Assessment Procedures for Utility Functions.” Management Science 28-8, 936-954.

Hershey, John C., and Paul J. H. Schoemaker (1985). "Probability versus Certainty

Equivalence Methods in Utility Measurement: Are They Equivalent?” Management Science 51-10. 
Holt, Charles A. (1986). "Preference Reversals and the Independence Axiom.” American Economic Review 76(3): 508-515.

Holt, Charles A., and Susan K. Laury (2002). "Risk Aversion and Incentive Effects in Lottery Choices.” American Economic Review 92(5): 1644-1655.

Holt, Charles A., and Susan K. Laury (2005). "Risk Aversion and Incentive Effects: New Data without Order Effects.” American Economic Review 95(3): 902-912.

Huber, Joel, John Payne and Christopher Puto (1982). “Adding Asymmetrically Dominated Alternatives. Violations of Regularity and the Similarity Hypothesis.” Journal of Consumer Research 9: 90-98.

Isaac, Mark, and Duncan James (2000). “Just Who Are You Calling Risk Averse?” Journal of Risk and Uncertainty 20(2): 177-187.

Lévy-Garboua, Louis, Hela Maafi, David Masclet and Antoine Terracol (2012). "Risk aversion and framing effects.” Experimental Economics 15: 128-144.

Machina, Mark J. (1982). “'Expected Utility' Analysis Without the Independence Axiom.” Econometrica 50(2): 227-323.

Machina, Mark J. (1983). “Generalized Expected Utility Analysis and the Nature of Observed Violations of the Independence Axiom." In B. Stigum and F. Wenstop, eds., Foundations of Utility and Risk Theory with Applications, Dordrecht: D. Reidel.

McCord, Mark, and Richard de Neufville (1986). "Lottery Equivalents: Reduction of the Certainty Effect Problem in Utility Assessment.” Management Science 32-1, 56-61.

Mas-Colell, Andreu, Michael Whinston, with Jerry Green (1995). Microeconomic Theory, Oxford: Oxford University Press.

Parducci, Allen, and Douglas H. Weddell (1986). "The Category Effect with Rating Scales: Number of Categories, Number of Stimuli and Method of Presentation.” Journal of Experimental Psychology: Human Perception and Performance 12(4): 496-516.

Robinson, Angela, Michael W. Jones-Lee and Graham Loomes (2001). "Visual Analog Scales, Standard Gambles and Relative Risk Aversion.” Medical Decision Making 21: 17-27.

Saha, Atanu (1993). "Expo-Power Utility: A Flexible Form for Absolute and Relative Risk Aversion.” American Journal of Agricultural Economics 75(4): 905-913.

Stewart, Neil, Gordon D. A. Brown and Nick Carter (2005). "Absolute Identification by Relative Judgment.” Psychological Review 112(4): 881-911.

Wakker, Peter (2010). Prospect Theory. Cambridge University Press, Cambridge, UK.

Wakker, Peter, and D. Deneffe (1996). "Eliciting von Neumann-Morgenstern Utilities when Probabilities are Distorted or Unknown.” Management Science 42, 1131-1150. 